\title{
Comparative Proteomic Profiling Reveals Molecular Characteristics Associated with Oogenesis and Oocyte Maturation during Ovarian Development of Bactrocera dorsalis (Hendel)
}

\author{
Dong Wei ${ }^{1,2,3}$ (D) Ran Li ${ }^{1,2}$, Meng-Yi Zhang ${ }^{1,2}$, Yu-Wei Liu ${ }^{1,2}$, Zheng Zhang ${ }^{1,2}$, \\ Guy Smagghe ${ }^{1,2,3, *}$ and Jin-Jun Wang ${ }^{1,2, *}$ \\ 1 Key Laboratory of Entomology and Pest Control Engineering, College of Plant Protection, \\ Southwest University, Chongqing 400716, China; dong_wei1988@yahoo.com (D.W.); \\ liran9610@163.com (R.L.); mengyi_zhang@yeah.net (M.-Y.Z.); yuwei1994@yahoo.com (Y.-W.L.); \\ zhangzheng9523@sina.com (Z.Z.) \\ 2 Academy of Agricultural Sciences, Southwest University, Chongqing 400716, China \\ 3 Department of Crop Protection, Ghent University, Ghent 9000, Belgium \\ * Correspondence: guy.smagghe@ugent.be (G.S.); wangjinjun@swu.edu.cn (J.-J.W.); \\ Tel.: +32-9-264-6150 (G.S.), +86-23-6825-0255 (J.-J.W.); Fax: +32-9-264-6239 (G.S.); +86-23-6825-1269 (J.-J.W.)
}

Received: 17 May 2017; Accepted: 24 June 2017; Published: 30 June 2017

\begin{abstract}
Time-dependent expression of proteins in ovary is important to understand oogenesis in insects. Here, we profiled the proteomes of developing ovaries from Bactrocera dorsalis (Hendel) to obtain information about ovarian development with particular emphasis on differentially expressed proteins (DEPs) involved in oogenesis. A total of 4838 proteins were identified with an average peptide number of 8.15 and sequence coverage of $20.79 \%$. Quantitative proteomic analysis showed that a total of 612 and 196 proteins were differentially expressed in developing and mature ovaries, respectively. Furthermore, 153, 196 and 59 potential target proteins were highly expressed in early, vitellogenic and mature ovaries and most tested DEPs had the similar trends consistent with the respective transcriptional profiles. These proteins were abundantly expressed in pre-vitellogenic and vitellogenic stages, including tropomyosin, vitellogenin, eukaryotic translation initiation factor, heat shock protein, importin protein, vitelline membrane protein, and chorion protein. Several hormone and signal pathway related proteins were also identified during ovarian development including piRNA, notch, insulin, juvenile, and ecdysone hormone signal pathways. This is the first report of a global ovary proteome of a tephritid fruit fly, and may contribute to understanding the complicate processes of ovarian development and exploring the potentially novel pest control targets.
\end{abstract}

Keywords: oriental fruit fly; isobaric tags for relative and absolute quantitation (iTRAQ); liquid chromatography-tandem mass spectrometry (LC-MS/MS); proteome; ovary; reproduction; oogenesis

\section{Introduction}

The oriental fruit fly, Bactrocera dorsalis (Hendel), is one of the most devastating agricultural pests worldwide due to its highly reproductive and invasive ability. Several factors that regulate the fecundity and mating behavior of $B$. dorsalis have been previously documented [1,2]. However, due to the long-term and frequent applications of chemical insecticides, $B$. dorsalis has evolved high levels of resistance to many commonly used insecticides [3-5]. Insecticide resistance poses a serious threat to current control effort for B. dorsalis and other tephritid flies, and there is a great need for novel, environmentally safe, and efficient technologies that are integrated with conventional chemical control 
methods for sustainable control of $B$. dorsalis. Developing novel pest control strategies can be facilitated by the knowledge of biology and genetics of $B$. dorsalis.

A number of databases with genetic information of $B$. dorsalis are now available such as the transcriptomes of various developmental stages that help identify genes involved in development and reproduction [6], as well as sex-determination [7]. Considerable efforts have also been devoted to creating a male-biased reproductive tissue-specific transcriptome to identify genes involved in spermatogenesis [8]. These genetic information can be served as sources for exploring new molecular targets for pest control; however, it is not always feasible to correlate the transcription levels of mRNAs with the synthesis of the corresponding proteins, and it is impossible to observe post-translational events such as protein modifications from gene expression studies. Therefore, it is necessary to identify proteins in developing testis/ovary directly rather than making inferences about their protein expression from the transcription profile. Thus far, little research has focused on the proteome of B. dorsalis for potential target identification. In male accessory glands, 90 proteins that likely regulate female reproduction were identified previously through proteomic approaches [9]. Although such transcriptomes of $B$. dorsalis are available at NCBI, large-scale molecular analysis of reproductive proteins expressed in $B$. dorsalis ovary and their abundance during the ovarian development remains unknown. Understanding the molecular mechanisms of ovary development and oogenesis is essential to manipulate female fertility.

Among the technologies, mass spectrometry has been widely used to analyze proteomes in reproductive tissues, such as testis, male accessory gland, ejaculatory duct, and ejaculatory bulb of Drosophila melanogaster [10]. Analysis of ovary proteome is required for a complete understanding of the physiological processes involved in oogenesis and ovary development. Global proteomic characterization of the D. melanogaster ovary was recently sequenced and analyzed [11], allowing the discovery of novel regulators and pathways. Such study also provides a systemic view of networks that govern ovarian pathophysiology and embryonic development in flies. In some aspects, Drosophila oogenesis represents a valuable developmental platform to genetically and morphologically dissect a wide range of biological processes, such as stem cell self-renewal [12], axis specification [13], cell differentiation and pattern formation [14].

It would be advantageous to identify ovarian proteins since they may have the potential to serve as targets for pest control. Targeting the ovary can be effective because it could affect reproduction by decreasing oogenesis and embryogenesis, thus leading to a reduction in the reproductive rate. Therefore, identification of molecular targets in B. dorsalis ovary may aid in the development of novel pest control methods that interfere with female reproduction. In this study, we performed a global proteome analysis of developing ovaries from B. dorsalis by using isobaric tags for relative and absolute quantitation (iTRAQ) labeling followed by liquid chromatography-tandem mass spectrometry (LC-MS/MS) with the aim to gather information about ovarian proteins with particular emphasis on differentially expressed proteins (DEPs) during development. The identification of these proteins will increase our understanding of ovarian and oocyte development, and aid in the identification of novel targets for improving tephritid fruit fly control strategies.

\section{Results and Discussion}

\subsection{Proteome-Wide Identification of Proteins in Ovaries}

We observed the ovarian morphology during development at different times (Figure 1), and found that the ovary develops to the vitellogenic stage at 5 days after emergency (Figure 1C). Also, the ovarian size changes significantly during the vitellogenic period. We identified 38,028 peptides, which were assembled to 4838 proteins from 1-, 6-, 9-day-old female ovaries (ov-1, ov-6 and ov-9, respectively) by using LC-MS/MS. Among these, identification of 4053 (83.77\%) proteins was based on the identity of more than one peptide. Most of the proteins were identified by 2-20 peptides; 1927 (39.83\%) proteins were identified by $2-5$ peptides, and $1070(22.12 \%)$ proteins by $6-10$ peptides (Figure $2 \mathrm{~A}$ ). About $3.45 \%$ 
of the proteins were identified by more than 30 peptides. The average peptide number of all proteins identified was 8.15 . The sequence coverage of specifically identified proteins was estimated as the percentage of matching amino acids between the identified peptides having more than 95\% confidence divided by the total number of amino acids in the protein sequence. The sequence coverage of 1813 $(37.47 \%)$ proteins was less than $10 \%$, and that of $1120(23.15 \%)$ was $10-20 \%$ (Figure $2 \mathrm{~B}$ ). The average coverage was $20.79 \%$, in which $15.94 \%$ were identified to have more than $40 \%$ sequence coverage.
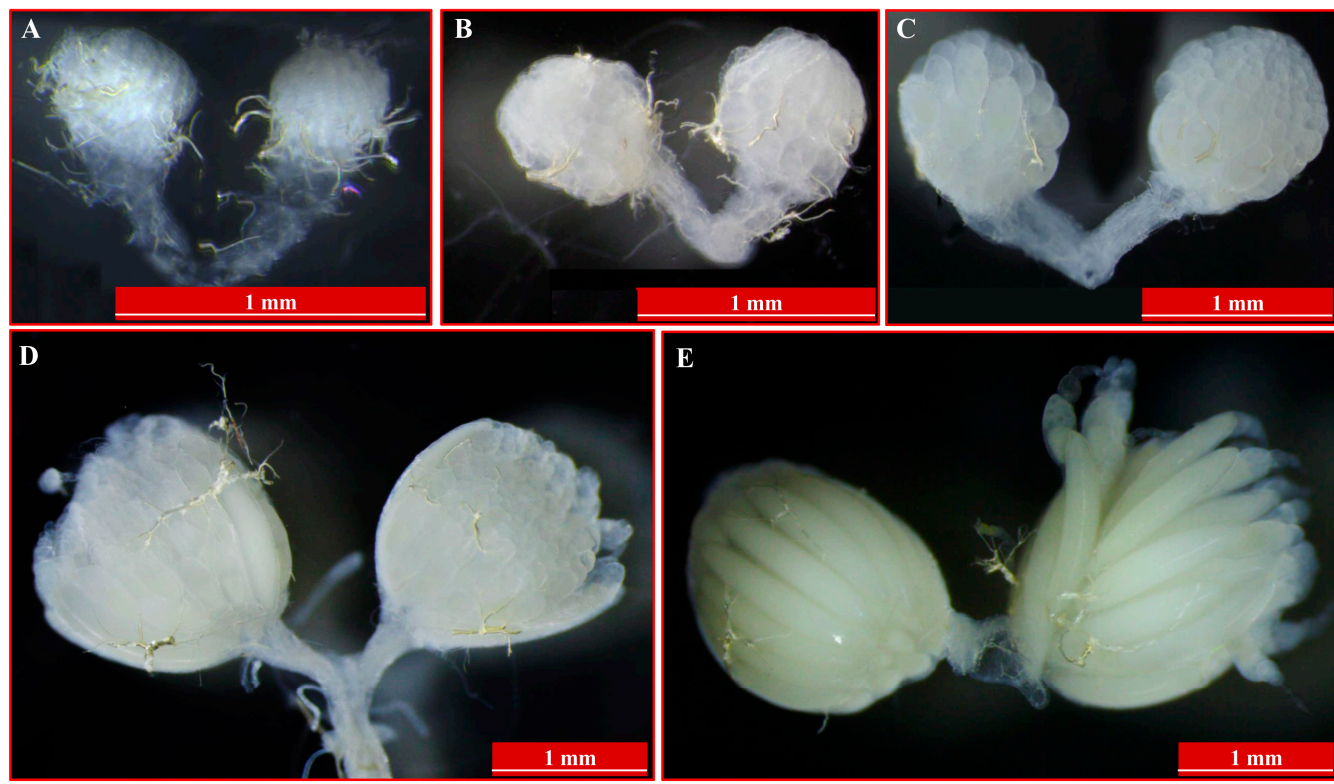

Figure 1. The images of ovaries at different development stages of Bactrocera dorsalis. Panels of (A-E) represent ovaries of 1-, 3-, 5-, 7-, and 9-day-old adults.
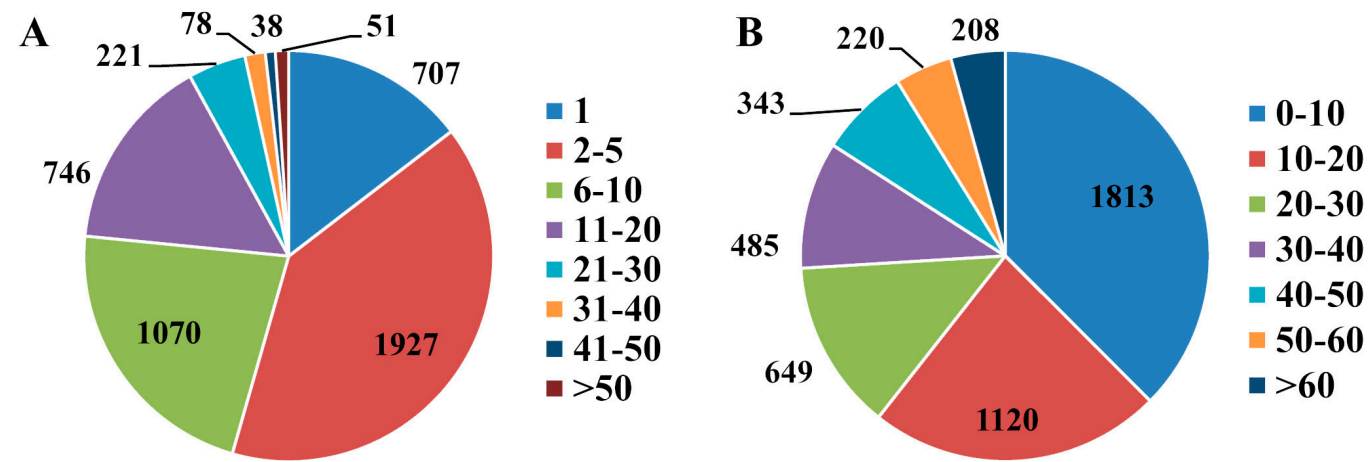

Figure 2. The distribution of peptide number (A) and sequence coverage (B) of proteins identified in ovaries of Bactrocera dorsalis during development.

\subsection{Functional Annotation of Proteins}

Because of the accessibility of the $B$. dorsalis genomic information, all identified proteins were transferred to UniProt. Among these identified proteins, 3758, 2401, and 2468 proteins were functionally annotated in Gene ontology (GO) and Cluster of Orthologous Groups (COG) databases, as well as the Kyoto Encyclopedia of Gene and Genomes (KEGG) pathway analysis, respectively. Then, 3758 proteins were categorized into 48 hierarchically structured GO classification, including three ontologies named biological process, cellular component, and molecular function. In the biological process ontology, "cellular progress" and "metabolic progress" were the most dominant categories containing $>2000$ proteins (Figure S1A). Many proteins involved in reproduction were identified in the biological process and included "reproduction", "reproductive process", and "viral reproduction". In the 
cellular component ontology, "cell" and "cell part" were the most predominant categories containing $>2000$ proteins (Figure S1B). However, in the molecular function ontology, the highly represented categories were "binding" and "catalytic activity," which contained $>1600$ proteins (Figure S1C). In the COG analysis, 2401 proteins were categorized into 24 COG classifications (Figure S1D). In addition to "general function prediction only," two categories of "posttranslational modification, protein turnover, chaperones" and "translation, ribosomal structure and biogenesis" were the most dominant categories, while the functions of 90 proteins were unknown. The pathway analysis by KEGG annotation showed that a total of 2468 proteins were mapped to 259 pathways in the KEGG database (Figure S1E). "Metabolic pathways" $(687,27.84 \%)$ and "biosynthesis of secondary metabolites" $(236,9.56 \%)$ were the largest two categories. There were 72 and 57 proteins assigned to "insulin signaling" and "oocyte meiosis" pathways respectively, that were functionally related with a specialized organ.

\subsection{Differentially Expressed Proteins}

In order to analyze the DEPs during the development of ovary, relative quantification of proteins was performed to analyze the abundance of proteins identified in three stages (Figure 3). Briefly, there were 612 DEPs abundant in "ov-1 vs. ov-6" comparison, 389 of which were highly expressed in ov-6 (Table S1), including chitinase-like proteins, heat shock proteins (Hsps), importin (Imp), eukaryotic translation initiation factors (eIFs), NADH dehydrogenases, piRNA pathway proteins, ribosomal proteins, and vitellogenins. Six chitinase-like proteins and three tropomyosins (Tpms) were down-regulated in ov-6. In the quantitative analysis of proteins, 104 and 92 DEPs were highly abundant in ov-9 and ov-6, respectively (Table S2). Three chorion related proteins were highly abundant in the mature ovary, while there were seven ATP-dependent RNA helicases, four eIFs, four ribosomal proteins, and four piRNA pathway proteins that were down-regulated in the mature ovary. Upon comparison with ovary of 1-day-old adult, 294 out of 512 DEPs were highly expressed in ov-9 (Table S3). Most of these DEPs (328) were also identified in the first quantitative comparison.

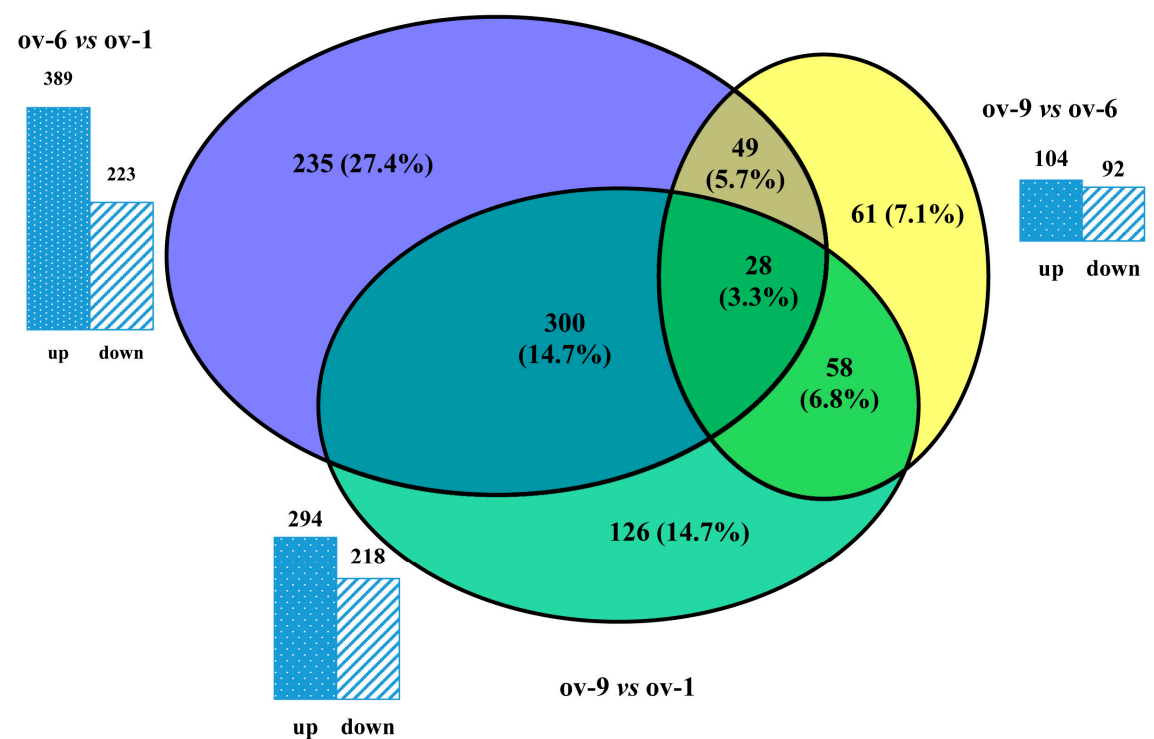

Figure 3. Venn diagrams of differentially expressed proteins between each of the developmental stages during ovary maturation of Bactrocera dorsalis. Circles are shown to scale but their overlaps are estimated in each diagram. Up and down mean the protein abundance in the later staged ovaries compared to the early staged ovaries. Number above the bar means the number of protein differentially expressed in two staged ovaries. Ov-1, ov-6 and ov-9 represent ovaries of 1-, 5- and 9-day-old adult of $B$. dorsalis. Different colors of blue, yellow and green represent the protein number of comparisons between ov-6 vs. ov-1, ov-9 vs. ov- 6 , and ov-9 vs. ov-1, respectively. 
All the DEPs between each comparison were functionally annotated as above. A total of 672 DEPs were functionally annotated into three ontologies by GO analysis (Figure 4), among which 419, 170, and 407 DEPs were involved in the biological process. The highly expressed categories were "cellular process", "metabolic process", and "multicellular organismal process" (Figure 4A). There were 249 and 231 DEPs involved in "reproduction" and "reproductive process," respectively. Moreover, "cell", "cell part" and "organelle" were the predominant categories in cellular component ontology (Figure 4B). In the molecular function category, most DEPs were assigned to "binding" and "catalytic activity" (Figure 4C). A total of 450 DEPs from each comparison were aligned to 24 categories after COG annotation (Figure 5). Most of the up-regulated DEPs in "ov-6 vs. ov-1" comparison were involved in "translation, ribosomal stricture and biogenesis" (70 DEPs), "posttranslational modification, protein turnover, chaperones" (56 DEPs), "energy production and conversion" (18 DEPs) and "general function prediction only" (50 DEPs). In the identification analysis, the largest category was "general functional prediction only" as mentioned above, indicating strong biogenesis and metabolism towards protein synthesis in ov-6. Twenty-nine DEPs involved in "translation, ribosomal stricture and biogenesis" were down-regulated in the mature ovary implying their roles specific to the vitellogenic stage.
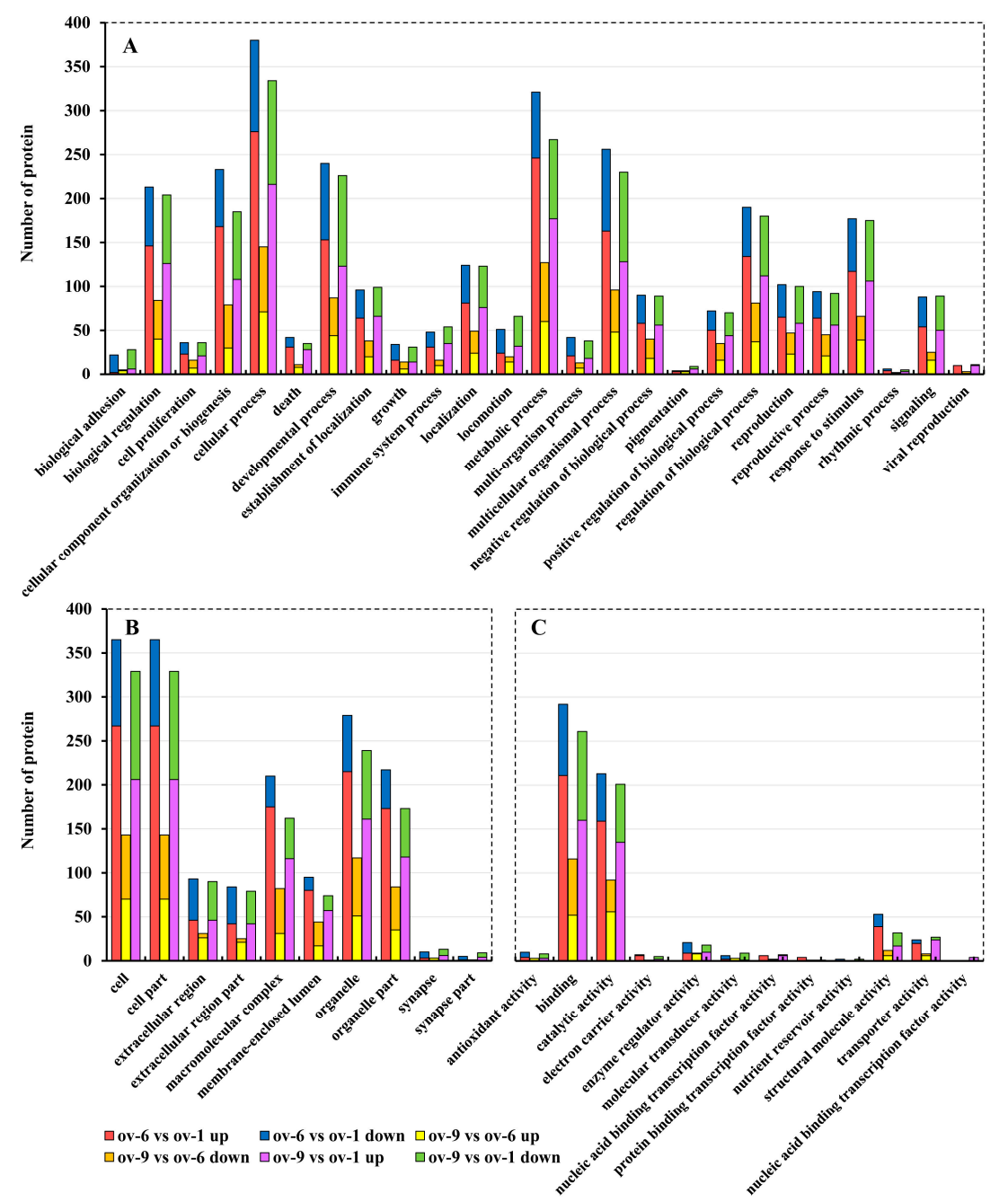

Figure 4. Gene ontology (GO) analysis of differentially expressed proteins in three comparisons by iTRAQ analysis. Shown above is the classification of these proteins in different categories based on biological process (A), cellular component (B), and molecular function (C). Ov-1, ov-6 and ov-9 represent ovaries of 1-, 6- and 9-day-old adult of B. dorsalis. 


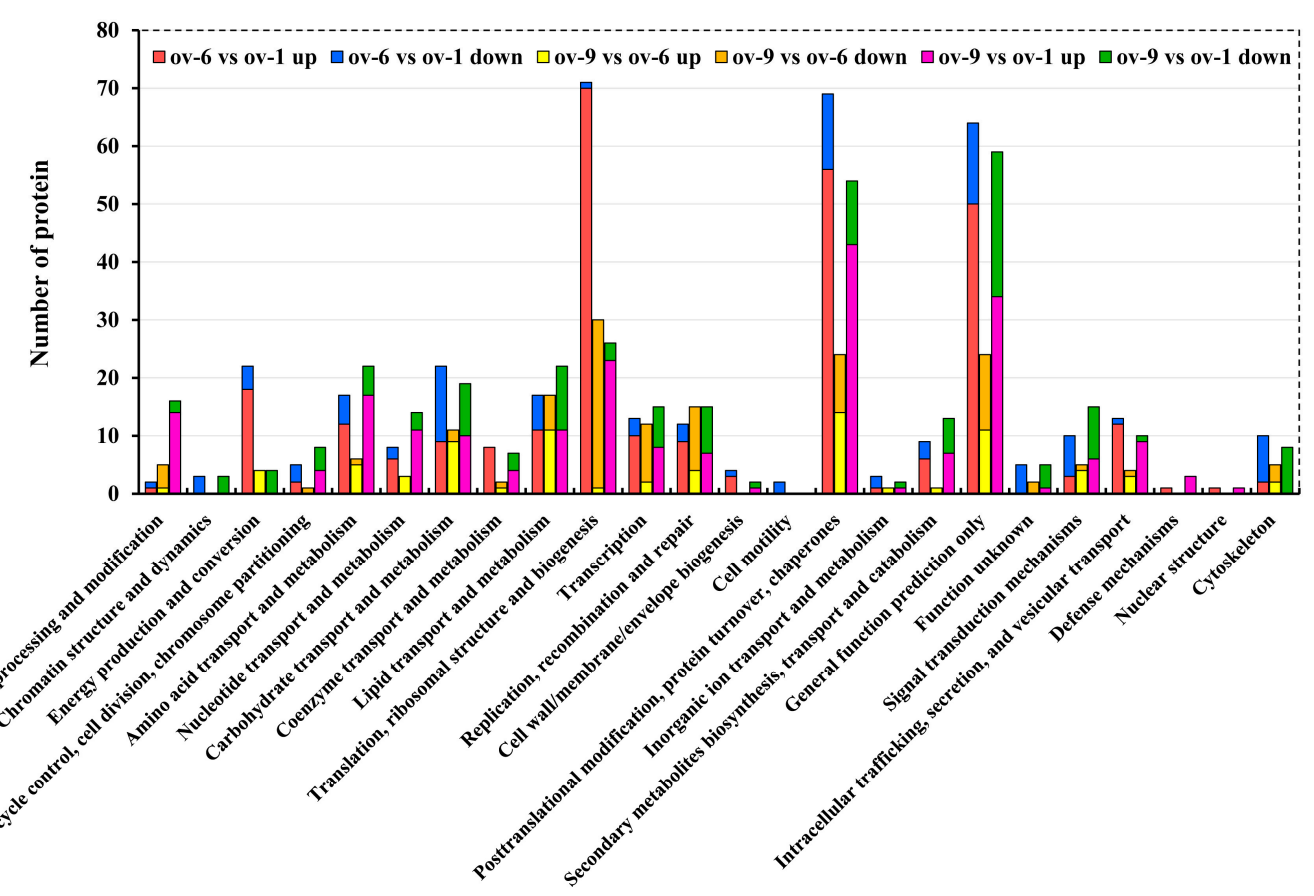

Figure 5. Clusters of Orthologous Groups of protein classification of differentially expressed proteins in three comparisons by iTRAQ analysis. Ov-1, ov-6 and ov-9 represent ovaries of 1-, 6- and 9-day-old adult of $B$. dorsalis.

\subsection{DEPs Highly Abundant Ovary of 1-Day-Old Adult}

A total of 153 DEPs were identified as highly abundant in ov-1 compared to later stages (Table 1), including tropomyosins (Tpms), myosin regulatory light chain, chitinase-like protein, cytochrome P450 (P450 4g15), development-specific protein, general odorant-binding protein (GOBP99a), glutathione $S$-transferase (GSTo1), and larval cuticle protein. Eight of these DEPs were validated at the transcriptional level by qRT-PCR. All eight genes showed high expression in 1-day-old ovary, which is consistent with the protein abundance (Figure 6). The high expression at both transcriptional and protein levels implied their crucial roles in the initial development of $B$. dorsalis ovary.

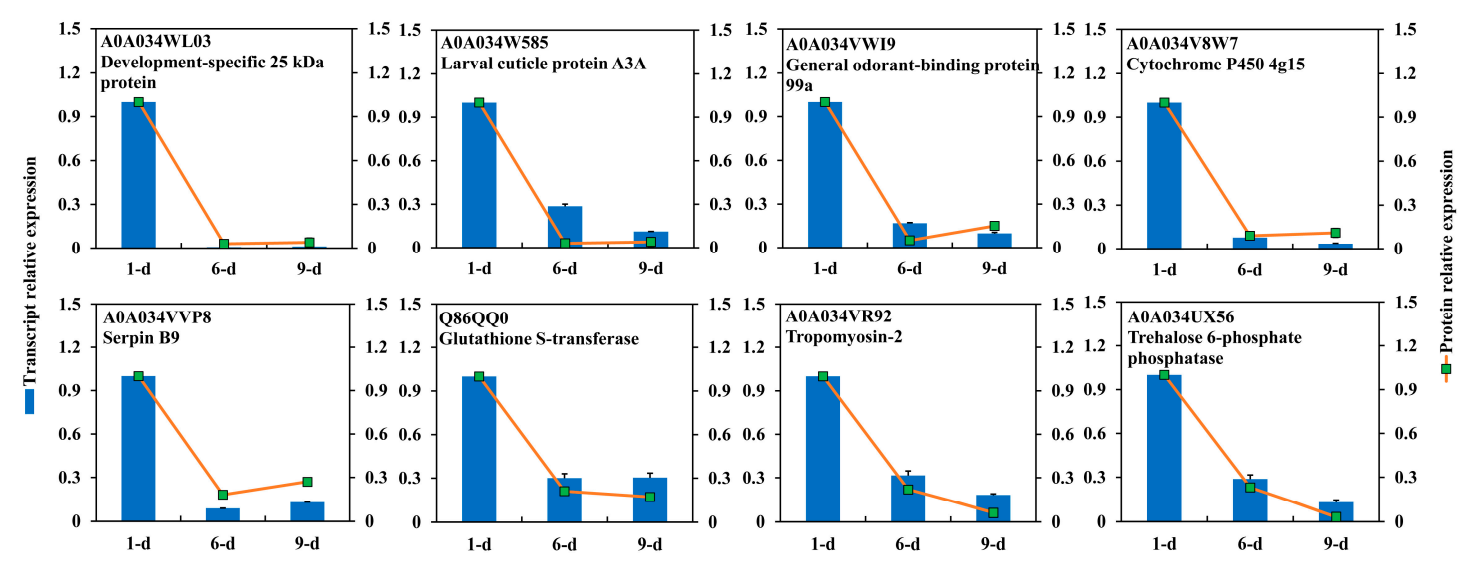

Figure 6. Transcriptional expression of proteins highly abundant in early ovary of 1-day-old Bactrocera dorsalis adult. Gene expression in three time-point stages of ovary, 1-day, 6-day and 9-day, were determined by quantitative PCR. All relative expression was compared to that in ovary of 1-day-old adult. 
Table 1. Proteins highly abundant in ovary of 1-day-old adult of Bactrocera dorsalis.

\begin{tabular}{|c|c|c|c|c|c|c|c|}
\hline Protein ID & Annotation & Length & Coverage & Peptides & Ov-6/Ov-1 & Ov-9/Ov-1 & Ov-9/Ov-6 ${ }^{a, b}$ \\
\hline A0A034WJQ4 & Fat-body protein 1 & 511 & 48.53 & 31 & $\downarrow 0.02 \pm 0$ & $\downarrow 0.02 \pm 0$ & $\uparrow 50.24 \pm 28.2^{2}$ \\
\hline A0A034V3B3 & Arylphorin subunit A4 & 1224 & 45.92 & 55 & $\downarrow 0.02 \pm 0$ & $\downarrow 0.04 \pm 0.01$ & $\uparrow 4.95 \pm 2.14^{\mathrm{a}}$ \\
\hline A0A034VYM4 & Larval serum protein 1 gamma chain & 773 & 31.31 & 27 & $\downarrow 0.02 \pm 0$ & $\downarrow 0.03 \pm 0$ & $\uparrow 2.95 \pm 1.03^{\mathrm{a}}$ \\
\hline A0A034V6N3 & High mobility group protein $\mathrm{Z}$ & 112 & 33.04 & 5 & $\downarrow 0.02 \pm 0$ & $\downarrow 0.02 \pm 0$ & $\uparrow 1.87 \pm 0.47^{\mathrm{a}}$ \\
\hline A0A034WL03 & Development-specific $25 \mathrm{kDa}$ protein & 259 & 69.5 & 19 & $\downarrow 0.03 \pm 0$ & $\downarrow 0.04 \pm 0$ & $\uparrow 3.04 \pm 1^{\mathrm{a}}$ \\
\hline A0A034W585 & Larval cuticle protein $\mathrm{A} 3 \mathrm{~A}$ & 138 & 75.36 & 17 & $\downarrow 0.03 \pm 0$ & $\downarrow 0.04 \pm 0$ & $\uparrow 2.3 \pm 0.55^{\mathrm{a}}$ \\
\hline A0A034WSW4 & Larval cuticle protein 8 & 104 & 22.12 & 2 & $\downarrow 0.03 \pm 0$ & $\downarrow 0.04 \pm 0.01$ & $\uparrow 1.98 \pm 0.23^{\mathrm{a}}$ \\
\hline A0A034WXX0 & Histone H1 & 240 & 30.83 & 11 & $\downarrow 0.03 \pm 0.01$ & $\downarrow 0.03 \pm 0$ & $\uparrow 8.18 \pm 4.64^{\mathrm{a}}$ \\
\hline A0A034WP07 & Attacin-A & 239 & 72.8 & 14 & $\downarrow 0.03 \pm 0.01$ & $\downarrow 0.13 \pm 0.03$ & $\uparrow 6.37 \pm 1.87^{\mathrm{a}}$ \\
\hline A0A034V3D6 & J domain-containing protein & 177 & 70.62 & 15 & $\downarrow 0.04 \pm 0.01$ & $\downarrow 0.03 \pm 0$ & $0.84 \pm 0.25^{b}$ \\
\hline A0A034W4Q0 & Larval cuticle protein A2B & 180 & 73.89 & 17 & $\downarrow 0.05 \pm 0$ & $\downarrow 0.06 \pm 0$ & $\uparrow 1.61 \pm 0.32^{\mathrm{a}}$ \\
\hline A0A034VT34 & Angiopoietin-4 & 454 & 20.04 & 7 & $\downarrow 0.05 \pm 0$ & $\downarrow 0.06 \pm 0.01$ & $1.39 \pm 0.36^{b}$ \\
\hline A0A034W9Y1 & Larval cuticle protein A2B & 250 & 92.4 & 19 & $\downarrow 0.05 \pm 0.01$ & $\downarrow 0.06 \pm 0$ & $\uparrow 1.73 \pm 0.6^{\mathrm{a}}$ \\
\hline A0A034VU29 & Regucalcin & 351 & 64.96 & 26 & $\downarrow 0.05 \pm 0.01$ & $\downarrow 0.05 \pm 0.01$ & $1.49 \pm 0.42^{b}$ \\
\hline $\mathrm{I} 1 \mathrm{~T} 1 \mathrm{H} 2$ & Heat shock protein 20 & 170 & 67.65 & 10 & $\downarrow 0.05 \pm 0.02$ & $\downarrow 0.13 \pm 0.03$ & $\uparrow 4.75 \pm 1.43^{\mathrm{a}}$ \\
\hline A0A034VSB5 & Fasciclin-1 & 542 & 35.42 & 17 & $\downarrow 0.07 \pm 0$ & $\downarrow 0.56 \pm 0.04$ & $\uparrow 8.35 \pm 0.24$ \\
\hline A0A034WRI9 & Sarcocystatin-A & 125 & 47.2 & 6 & $\downarrow 0.07 \pm 0.02$ & $\downarrow 0.15 \pm 0.04$ & $\uparrow 3.48 \pm 1.58^{\mathrm{a}}$ \\
\hline A0A034WF27 & TPPP family protein CG4893 & 201 & 40.3 & 7 & $\downarrow 0.08 \pm 0.01$ & $\downarrow 0.09 \pm 0.02$ & $1.12 \pm 0.28^{b}$ \\
\hline A0A034VUV3 & Lamin Dm0 & 617 & 71.64 & 46 & $\downarrow 0.08 \pm 0.02$ & $\downarrow 0.06 \pm 0$ & $0.8 \pm 0.28^{b}$ \\
\hline A0A034WSV2 & Lamin-C & 622 & 56.27 & 37 & $\downarrow 0.09 \pm 0.01$ & $\downarrow 0.06 \pm 0$ & $\downarrow 0.63 \pm 0.13^{\mathrm{a}}$ \\
\hline A0A034V8W7 & Cytochrome P450 4g15 & 484 & 23.76 & 10 & $\downarrow 0.09 \pm 0.01$ & $\downarrow 0.11 \pm 0.03$ & $1.27 \pm 0.29 \mathrm{~b}$ \\
\hline A0A034VPE5 & Tropomyosin-1, isoforms 9A/A/B & 188 & 69.68 & 19 & $\downarrow 0.09 \pm 0.01$ & $\downarrow 0.1 \pm 0.02$ & $0.92 \pm 0.09 \mathrm{~b}$ \\
\hline A0A034V2U0 & Protein 4.1 -like protein & 1703 & 57.37 & 82 & $\downarrow 0.09 \pm 0.02$ & $\downarrow 0.07 \pm 0$ & $0.96 \pm 0.24^{b}$ \\
\hline A0A034VW09 & Protein Skeletor & 741 & 29.82 & 14 & $\downarrow 0.1 \pm 0.01$ & $\downarrow 0.21 \pm 0.05$ & $\uparrow 2.25 \pm 0.46^{\mathrm{a}}$ \\
\hline A0A034W3W2 & Regucalcin & 296 & 42.91 & 12 & $\downarrow 0.1 \pm 0.02$ & $\downarrow 0.08 \pm 0.01$ & $0.87 \pm 0.25^{b}$ \\
\hline A0A034V7Q2 & Protein yellow & 261 & 31.42 & 6 & $\downarrow 0.1 \pm 0.03$ & $\downarrow 0.13 \pm 0.01$ & $1.48 \pm 0.41^{b}$ \\
\hline A0A034VI82 & Tropomodulin & 386 & 34.97 & 10 & $\downarrow 0.11 \pm 0$ & $\downarrow 0.08 \pm 0$ & $\downarrow 0.62 \pm 0.07^{\mathrm{a}}$ \\
\hline A0A034VUS5 & Integrin-linked protein kinase & 448 & 32.37 & 14 & $\downarrow 0.12 \pm 0.01$ & $\downarrow 0.13 \pm 0.02$ & $1.15 \pm 0.3^{b}$ \\
\hline A0A034VKK9 & Alcohol dehydrogenase & 324 & 38.89 & 13 & $\downarrow 0.12 \pm 0.01$ & $\downarrow 0.13 \pm 0.02$ & $0.94 \pm 0.2^{b}$ \\
\hline A0A034VYW9 & Contactin & 1393 & 25.2 & 27 & $\downarrow 0.12 \pm 0.02$ & $\downarrow 0.21 \pm 0.04$ & $\uparrow 2.08 \pm 0.42^{\mathrm{a}}$ \\
\hline A0A034WVT6 & Membrane metallo-endopeptidase-like 1 & 714 & 22.13 & 14 & $\downarrow 0.12 \pm 0.02$ & $\downarrow 0.15 \pm 0.01$ & $1.18 \pm 0.19^{b}$ \\
\hline A0A034V5Q1 & Ras suppressor protein 1 & 138 & 39.86 & 5 & $\downarrow 0.13 \pm 0.02$ & $\downarrow 0.19 \pm 0.03$ & $\uparrow 1.67 \pm 0.41^{\mathrm{a}}$ \\
\hline A0A034W0B4 & Chitinase-like protein CG5210 & 279 & 51.25 & 14 & $\downarrow 0.13 \pm 0.04$ & $\downarrow 0.37 \pm 0.04$ & $\uparrow 4.97 \pm 1.76^{\mathrm{a}}$ \\
\hline A0A034WBA7 & Putative G-protein coupled receptor 158 & 809 & 29.05 & 20 & $\downarrow 0.14 \pm 0.02$ & $\downarrow 0.28 \pm 0.04$ & $\uparrow 2.24 \pm 0.56^{\mathrm{a}}$ \\
\hline
\end{tabular}


Table 1. Cont.

\begin{tabular}{|c|c|c|c|c|c|c|c|}
\hline Protein ID & Annotation & Length & Coverage & Peptides & Ov-6/Ov-1 & Ov-9/Ov-1 & Ov-9/Ov-6 ${ }^{a, b}$ \\
\hline A0A034V5R2 & Talin-1 & 1928 & 48.86 & 69 & $\downarrow 0.15 \pm 0.01$ & $\downarrow 0.45 \pm 0.02$ & $\uparrow 3.15 \pm 0.09$ \\
\hline A0A034VQZ0 & Zinc finger protein 512B & 427 & 26.23 & 7 & $\downarrow 0.15 \pm 0.01$ & $\downarrow 0.13 \pm 0.02$ & $0.84 \pm 0.16^{b}$ \\
\hline A0A034VDB5 & Protein lap4 & 915 & 20.11 & 14 & $\downarrow 0.16 \pm 0$ & $\downarrow 0.11 \pm 0.01$ & $\downarrow 0.6 \pm 0.09^{\mathrm{a}}$ \\
\hline A0A034VTK3 & Chitinase-like protein Idgf5 & 432 & 27.31 & 9 & $\downarrow 0.16 \pm 0.05$ & $\downarrow 0.16 \pm 0.01$ & $1.27 \pm 0.41^{b}$ \\
\hline A0A034W8P6 & Protein E(Sev)2B & 211 & 43.6 & 9 & $\downarrow 0.17 \pm 0.03$ & $\downarrow 0.29 \pm 0.02$ & $\uparrow 2.05 \pm 0.44^{\mathrm{a}}$ \\
\hline A0A034VVP8 & Serpin B9 & 504 & 29.76 & 14 & $\downarrow 0.18 \pm 0.01$ & $\downarrow 0.27 \pm 0.02$ & $1.49 \pm 0.12^{b}$ \\
\hline A0A034W4R7 & $\alpha, \alpha$-trehalose-phosphate synthase A & 813 & 33.46 & 24 & $\downarrow 0.18 \pm 0.02$ & $\downarrow 0.09 \pm 0.01$ & $\downarrow 0.41 \pm 0.06^{\mathrm{a}}$ \\
\hline A0A034WI29 & Protein lin-7-like protein B & 195 & 28.72 & 5 & $\downarrow 0.18 \pm 0.03$ & $\downarrow 0.19 \pm 0.02$ & $1.1 \pm 0.18^{b}$ \\
\hline A0A034VY88 & Protein hu-li tai shao & 698 & 69.63 & 35 & $\downarrow 0.19 \pm 0.01$ & $\downarrow 0.2 \pm 0.02$ & $1.07 \pm 0.09^{b}$ \\
\hline A0A034W1X2 & Alaserpin & 398 & 27.39 & 10 & $\downarrow 0.19 \pm 0.02$ & $\downarrow 0.42 \pm 0.03$ & $\uparrow 2.43 \pm 0.28^{\mathrm{a}}$ \\
\hline A0A034W8L1 & Flotillin-2 & 425 & 43.53 & 17 & $\downarrow 0.19 \pm 0.02$ & $\downarrow 0.13 \pm 0.02$ & $\downarrow 0.63 \pm 0.08^{\mathrm{a}}$ \\
\hline A0A034VQR8 & Troponin T, skeletal muscle & 384 & 39.32 & 22 & $\downarrow 0.19 \pm 0.02$ & $\downarrow 0.07 \pm 0.01$ & $\downarrow 0.31 \pm 0.07^{\mathrm{a}}$ \\
\hline A0A034WRQ2 & $\alpha$-parvin & 366 & 33.06 & 10 & $\downarrow 0.19 \pm 0.05$ & $\downarrow 0.26 \pm 0.05$ & $\uparrow 1.58 \pm 0.39^{\mathrm{a}}$ \\
\hline A0A034VP83 & Poly(U)-specific endoribonuclease-like protein & 675 & 24.74 & 13 & $\downarrow 0.2 \pm 0.01$ & $\downarrow 0.35 \pm 0.03$ & $\uparrow 1.79 \pm 0.16^{\mathrm{a}}$ \\
\hline A0A034WSM6 & Guanine nucleotide-binding protein $G(S)$ subunit $\alpha$ & 382 & 32.2 & 10 & $\downarrow 0.2 \pm 0.02$ & $\downarrow 0.36 \pm 0.02$ & $\uparrow 1.88 \pm 0.18^{\mathrm{a}}$ \\
\hline Q86QQ0 & Glutathione S-transferase & 209 & 37.32 & 6 & $\downarrow 0.21 \pm 0.01$ & $\downarrow 0.17 \pm 0.01$ & $0.81 \pm 0.07^{b}$ \\
\hline A0A034VND6 & Tropomyosin-1, isoforms 33/34 & 282 & 48.58 & 20 & $\downarrow 0.21 \pm 0.04$ & $\downarrow 0.13 \pm 0.02$ & $\downarrow 0.61 \pm 0.13^{\mathrm{a}}$ \\
\hline A0A034VSX0 & Phosphate-regulating neutral endopeptidase & 683 & 22.69 & 13 & $\downarrow 0.21 \pm 0.04$ & $\downarrow 0.16 \pm 0.02$ & $0.85 \pm 0.16^{b}$ \\
\hline A0A034V6M3 & $60 S$ ribosomal protein L23a & 266 & 26.32 & 10 & $\downarrow 0.21 \pm 0.05$ & $\downarrow 0.26 \pm 0.07$ & $\uparrow 1.62 \pm 0.78^{a}$ \\
\hline A0A034UZS9 & PDZ and LIM domain protein & 495 & 22.22 & 9 & $\downarrow 0.22 \pm 0.01$ & $\downarrow 0.19 \pm 0.04$ & $0.87 \pm 0.25^{b}$ \\
\hline A0A034VR92 & Tropomyosin-2 & 284 & 57.04 & 17 & $\downarrow 0.22 \pm 0.02$ & $\downarrow 0.06 \pm 0$ & $\downarrow 0.22 \pm 0.02$ \\
\hline A0A034V2K7 & Spectrin $\beta$ chain, non-erythrocytic 5 & 4200 & 26.48 & 94 & $\downarrow 0.22 \pm 0.02$ & $\downarrow 0.29 \pm 0.03$ & $1.32 \pm 0.04^{b}$ \\
\hline A0A034VDP0 & Obscurin & 631 & 30.74 & 13 & $\downarrow 0.22 \pm 0.03$ & $\downarrow 0.15 \pm 0.02$ & $0.71 \pm 0.19^{b}$ \\
\hline A0A034UX56 & Trehalose 6-phosphate phosphatase & 274 & 81.75 & 25 & $\downarrow 0.23 \pm 0.01$ & $\downarrow 0.03 \pm 0$ & $\downarrow 0.08 \pm 0.01$ \\
\hline A0A034VE41 & Twitchin & 4978 & 23.54 & 86 & $\downarrow 0.23 \pm 0.02$ & $\downarrow 0.14 \pm 0.01$ & $\downarrow 0.57 \pm 0.05^{\mathrm{a}}$ \\
\hline A0A034WWI9 & Ejaculatory bulb-specific protein 3 & 127 & 22.05 & 3 & $\downarrow 0.23 \pm 0.02$ & $\downarrow 0.18 \pm 0.04$ & $0.77 \pm 0.14^{b}$ \\
\hline A0A034VPA5 & Heterogeneous nuclear ribonucleoprotein Q & 417 & 21.34 & 9 & $\downarrow 0.23 \pm 0.05$ & $\downarrow 0.15 \pm 0.01$ & $0.69 \pm 0.15^{b}$ \\
\hline A0A034VWH3 & Myosin regulatory light chain 2 & 222 & 52.7 & 10 & $\downarrow 0.25 \pm 0.02$ & $\downarrow 0.12 \pm 0.02$ & $\downarrow 0.41 \pm 0.06^{\mathrm{a}}$ \\
\hline A0A034VAC6 & Protein elav & 446 & 35.65 & 12 & $\downarrow 0.26 \pm 0.02$ & $\downarrow 0.29 \pm 0.04$ & $1.08 \pm 0.17^{b}$ \\
\hline A0A034V4T6 & Fasciclin-2 & 520 & 45.19 & 17 & $\downarrow 0.26 \pm 0.03$ & $\downarrow 0.08 \pm 0.01$ & $\downarrow 0.27 \pm 0.02^{\mathrm{a}}$ \\
\hline A0A034VRQ6 & Protein held out wings & 356 & 37.64 & 11 & $\downarrow 0.28 \pm 0.01$ & $\downarrow 0.15 \pm 0.01$ & $\downarrow 0.48 \pm 0.02^{\mathrm{a}}$ \\
\hline A0A034WML8 & Protein takeout & 269 & 20.07 & 4 & $\downarrow 0.29 \pm 0.02$ & $\downarrow 0.14 \pm 0.01$ & $\downarrow 0.46 \pm 0.05^{\mathrm{a}}$ \\
\hline A0A034WF58 & Glutathione peroxidase & 278 & 48.92 & 13 & $\downarrow 0.29 \pm 0.05$ & $\downarrow 0.26 \pm 0.03$ & $0.94 \pm 0.18^{b}$ \\
\hline A0A034VTK4 & DNA topoisomerase 2 & 1481 & 42.13 & 59 & $\downarrow 0.3 \pm 0.02$ & $\downarrow 0.24 \pm 0.01$ & $0.76 \pm 0.06^{b}$ \\
\hline A0A034WS42 & Histone $\mathrm{H} 4$ & 103 & 65.05 & 15 & $\downarrow 0.3 \pm 0.06$ & $\downarrow 0.08 \pm 0.01$ & $\downarrow 0.24 \pm 0.05$ \\
\hline
\end{tabular}


Table 1. Cont.

\begin{tabular}{|c|c|c|c|c|c|c|c|}
\hline Protein ID & Annotation & Length & Coverage & Peptides & Ov-6/Ov-1 & Ov-9/Ov-1 & Ov-9/Ov-6 ${ }^{a, b}$ \\
\hline A0A034WN98 & $17-\beta$-hydroxysteroid dehydrogenase 13 & 326 & 25.15 & 7 & $\downarrow 0.33 \pm 0.05$ & $\downarrow 0.3 \pm 0.07$ & $0.98 \pm 0.27^{b}$ \\
\hline A0A034V7N7 & Protein lethal(2) giant larvae & 1176 & 24.23 & 21 & $\downarrow 0.33 \pm 0.05$ & $\downarrow 0.24 \pm 0.04$ & $0.67 \pm 0.11^{b}$ \\
\hline M1F3Z9 & Glutathione S-transferase $\omega-1$ & 255 & 46.67 & 11 & $\downarrow 0.34 \pm 0.02$ & $\downarrow 0.26 \pm 0.02$ & $0.76 \pm 0.06^{b}$ \\
\hline A0A034VDX0 & L-2-hydroxyglutarate dehydrogenase, mitochondrial & 455 & 31.21 & 10 & $\downarrow 0.35 \pm 0.03$ & $\downarrow 0.33 \pm 0.07$ & $0.95 \pm 0.23^{b}$ \\
\hline A0A034VM61 & Clavesin-2 & 320 & 33.44 & 9 & $\downarrow 0.35 \pm 0.04$ & $\downarrow 0.45 \pm 0.07$ & $1.24 \pm 0.16^{\mathrm{b}}$ \\
\hline A0A034VPR7 & Putative peptidyl-prolyl cis-trans isomerase dodo & 160 & 57.5 & 11 & $\downarrow 0.35 \pm 0.06$ & $\downarrow 0.32 \pm 0$ & $0.99 \pm 0.18^{b}$ \\
\hline A0A034WSZ7 & Heterochromatin protein 1 & 212 & 23.11 & 5 & $\downarrow 0.37 \pm 0.02$ & $\downarrow 0.24 \pm 0.01$ & $\downarrow 0.6 \pm 0.01^{\mathrm{a}}$ \\
\hline A0A034WQQ0 & Calcyphosin-like protein & 219 & 27.4 & 6 & $\downarrow 0.37 \pm 0.1$ & $\downarrow 0.35 \pm 0.06$ & $1.04 \pm 0.21^{b}$ \\
\hline A0A034WBM1 & Acetyl-CoA acetyltransferase, cytosolic & 394 & 41.62 & 11 & $\downarrow 0.41 \pm 0.08$ & $\downarrow 0.45 \pm 0.05$ & $1.24 \pm 0.3^{b}$ \\
\hline A0A034VYI5 & Non-specific protein-tyrosine kinase & 510 & 27.25 & 12 & $\downarrow 0.45 \pm 0.07$ & $\downarrow 0.34 \pm 0.01$ & $0.79 \pm 0.12^{b}$ \\
\hline A0A034VW38 & SUN domain-containing protein 1 & 594 & 21.55 & 13 & $\downarrow 0.45 \pm 0.1$ & $\downarrow 0.31 \pm 0.09$ & $0.7 \pm 0.14^{b}$ \\
\hline A0A034VQG5 & Nucleoprotein TPR & 2410 & 26.22 & 53 & $\downarrow 0.47 \pm 0.03$ & $\downarrow 0.26 \pm 0.01$ & $\downarrow 0.49 \pm 0.05^{\mathrm{a}}$ \\
\hline A0A034VKE6 & $\begin{array}{l}\text { Bifunctional methylenetetrahydrofolate } \\
\text { dehydrogenase/cyclohydrolase, mitochondrial }\end{array}$ & 316 & 32.59 & 8 & $\downarrow 0.5 \pm 0.01$ & $\downarrow 0.21 \pm 0.05$ & $\downarrow 0.43 \pm 0.08^{\mathrm{a}}$ \\
\hline A0A034VPM4 & Vinculin & 960 & 50.63 & 38 & $\downarrow 0.54 \pm 0.04$ & $\downarrow 0.54 \pm 0.04$ & $0.98 \pm 0.07^{b}$ \\
\hline A0A034VRI7 & $\alpha$-actinin, sarcomeric & 895 & 62.35 & 53 & $\downarrow 0.55 \pm 0.04$ & $\downarrow 0.42 \pm 0.02$ & $0.75 \pm 0.04^{b}$ \\
\hline
\end{tabular}


Tpms are actin-binding cytoskeletal proteins, which play vital roles in various cellular processes including cytokinesis, cell migration, embryogenesis, and oocyte maturation $[15,16]$. Several Tpms isoforms were found to be expressed in specific developmental patterns, correlating with the differentiation of embryonic stem cells and response to polarizing signals at early embryonic development in the mouse ovary $[15,17]$. Tpm3 localizes in the cortex before metaphase II of the mouse oocyte, showing a role in asymmetric cell division and maintenance of cortical integrity [16]. In Drosophila, five Tpms are identified, and Tpm2 plays a role in oskar RNA localization in the posterior pole of oocytes resulting in the development of the abdomen and germ line [18]. Lack of Tpm2 affects head morphogenesis leading to death at the first instar stage [19]. In this study, three Tpms were identified, namely Tpm1 (A0A034VND6, A0A034VPE5) and Tpm2 (A0A034VR92), which showed high abundance in ov-1. The transcriptional expression of Tpm2 showed a consistent pattern with the proteomic data, indicating a similar role in oogenesis and ovarian development in $B$. dorsalis.

Dramatic change in the localization of cytoplasmic myosin (non-muscle) is a characteristic feature of early embryogenesis in D. melanogaster [20]. Maternal supply of myosin II is required for cytoplasmic transport during oogenesis [21]. Germ line cystoblasts lacking a functional light chain myosin II show severe defects in proliferation and cytokinesis. For instance, the cytoplasmic bridges linking the oocyte to the nurse cells in the egg chamber are abnormal during oogenesis [22,23]. In addition, numerous myosin heavy chain accumulate in the light chain deficient cells. Similar to Drosophila, a non-muscle light chain myosin was identified as abundant in ov-1. The essential role of myosin II for rapid cytoplasmic transport during oogenesis was also investigated [21]. A myosin V is confirmed to regulate oskar mRNA localization in the Drosophila oocyte [24], and an unconventional myosin VI, encoded by myosin heavy chain at 95F, is required for follicle cell epithelial development during egg chamber morphogenesis [25]. Both homologous myosin V (A0A034V675) and myosin VI (A0A034V7N4) were also identified to be highly abundant in ov-6.

There were many proteins that were highly abundant in the initial stage of $B$. dorsalis ovary including GOBP, GSTs, P450, development-specific protein, notch-like protein, serine/threonine protein kinase and its inhibitor (Serpin), and metalloproteases. It has been reported that chemosensory proteins are abundantly expressed in the female reproductive organ [26], although their function in reproduction has been studied mostly in male insects [27]. Formation of notch signaling regulation, maintenance of the germ stem cell niche, and the role of cap cells in determining the niche size in the Drosophila ovary has been well studied [28,29]. GSTs expressed in the reproductive organ have been identified in testis germ cells of male rats, and their activity increased after exposure to oxidative stress [30]. Transcriptional expression showed that one GST (Q86QQ0) was highly expressed in the early stage of ovary. Additionally, another detoxifying enzyme, P450, was also identified to be abundant in ov-1. The specific functions of these DEPs should be determined prior to their use for pest control. In addition, a development-specific protein (A0A034WL03), and SerpinB9 (A0A034VVP8) were abundantly present in ov-1, indicating their stage specific function in oogenesis. Interestingly, four larval cuticle proteins and three chitinase-like proteins were identified as abundant in ov-1, but their functions in ovarian development are not known. Twenty seven uncharacterized proteins were identified during the initial stage of ovary. These proteins likely play roles in cell differentiation during early ovarian development. A large number of proteins (48\%) in newly formed ovaries were also not functionally identified in Metapenaeus ensis [31]. Further studies should focus on the early development of insect ovary.

\subsection{DEPs Highly Abundant in Ovary of 6-Day-Old Adult}

Among the DEPs, 389 were up-regulated during intermediate stages of ovary. Of these, 44 were down-regulated at mature ovary (Table 2), including eIFs, Imps, ribosomal proteins, villin-like protein, as well as piRNA pathway related proteins. Additionally, 152 proteins highly abundant in ov- 6 were also found to be highly expressed in ov-9 (Table S4), such as vitelline membrane protein (Vm26Aa), vitellogenin (Vg1), GOBP99b, P450 306a1, Hsps (Hsp70, Hsp75, Hsp60, and Hsp23), 26S proteasome, 
and GST, etc. Transcriptional expressions of tested proteins differed from the protein expression (Figure 7). Three out of six DEPs abundant in ov-6 were highly expressed in the mature ovary with only one DEP (prolyl 4-hydroxylase $\alpha 2$ ) highly expressed in ov- 6 at transcriptional levels. Two out of six DEPs abundant in both vitellogenic and mature ovaries were highly expressed in ov-6, namely Vm26Aa and GOBP99b; two DEPs were highly expressed in mature ovary at the transcriptional level; and two DEPs had the same expression patterns.
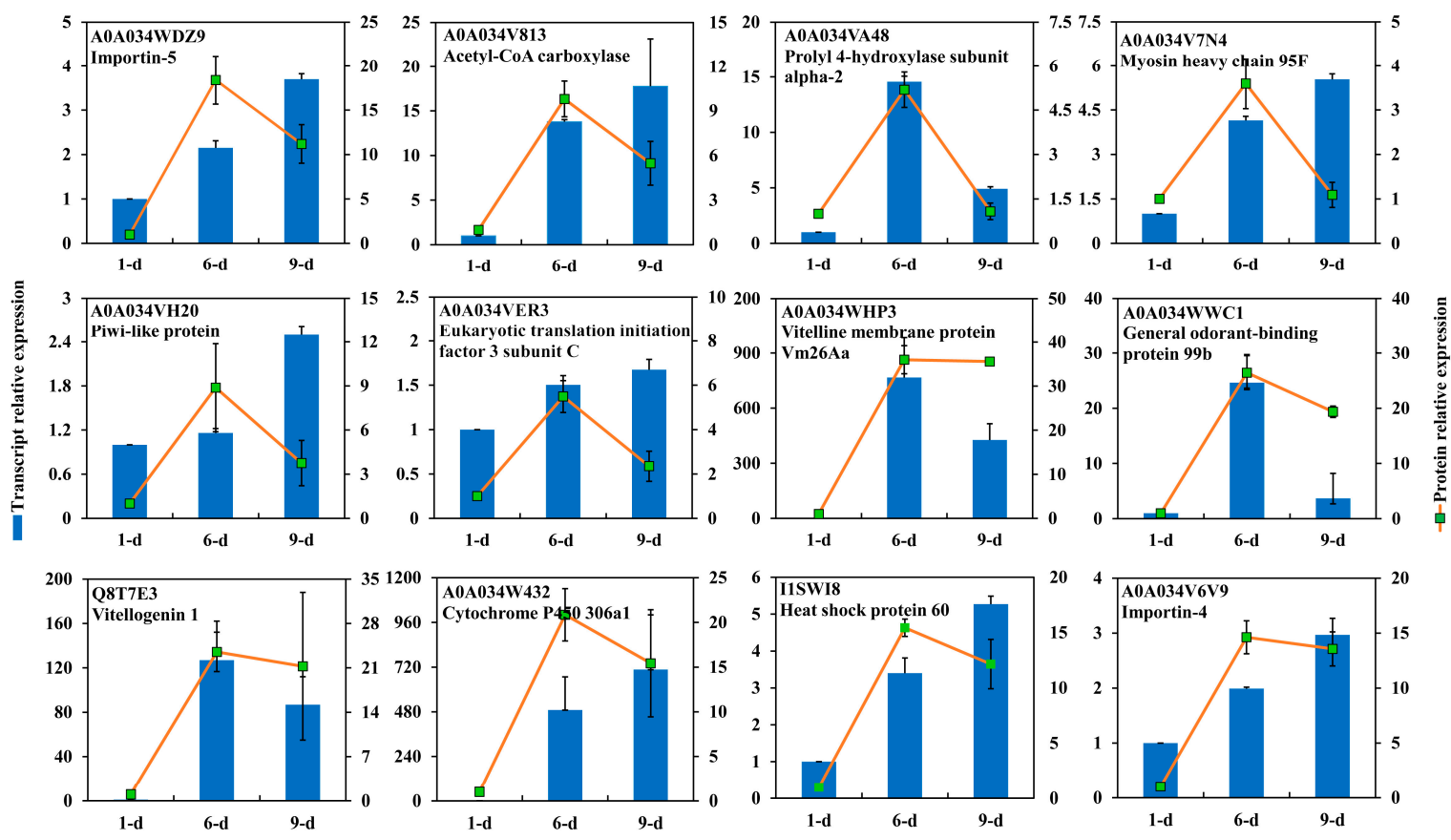

Figure 7. Transcriptional expression of proteins highly abundant in ovary of 6-day-old Bactrocera dorsalis adult. Gene expression was calculated as Figure 6.

In Drosophila, there are three Piwi proteins termed Aubergine, Piwi, and Argonaute- 3 in distinct piRNA pathways with different functions in ovarian germ and somatic cells development [32]. Two interrelated branches of the piRNA system have been reported: somatic cells that support oogenesis only employ Piwi, whereas germ cells utilize a more elaborate pathway centered on the three gonad-specific Argonaute proteins [32,33]. Piwi protein regulates both niche and intrinsic mechanisms to maintain germline stem cells during oogenesis in Drosophila [34]. Also, Tudor protein in Drosophila is a component of two types of germ granules: nuage, which is assembled during ovary development; and polar granule, which forms at the posterior cytoplasm of the oocyte and is maintained in early embryo [35]. A novel role was recently reported for Tudor domains in the Tudor-Aubergine protein complex assembly and distribution during Drosophila oogenesis [36]. It has also been demonstrated that some of the Piwi proteins are necessary for very early stages of oogenesis within the germarium [37]. The complex regulation of piRNA pathway on oogenesis is clear in Drosophila, showing a potential use in pest control by regulating reproduction. A piwi-like protein and two tudor-domain-containing proteins were identified to be highly expressed in ov-6 likely due to their regulation in oocyte maturation. 
Table 2. Proteins highly abundant in ovary of 6-d-old adult of Bactrocera dorsalis.

\begin{tabular}{|c|c|c|c|c|c|c|c|}
\hline Protein ID & Annotation & Length & Coverage & Peptides & Ov-6/Ov-1 & Ov-9/Ov-1 ${ }^{a, b}$ & Ov-9/Ov-6 \\
\hline A0A034V9A3 & CUGBP Elav-like family member 2 & 587 & 25.55 & 13 & $\uparrow 29.06 \pm 4.88$ & $\uparrow 13.14 \pm 6.66^{\mathrm{a}}$ & $\downarrow 0.37 \pm 0.06$ \\
\hline A0A034WDZ9 & Importin-5 & 1106 & 53.35 & 47 & $\uparrow 18.4 \pm 2.73$ & $\uparrow 11.19 \pm 4.34$ & $\downarrow 0.53 \pm 0.04$ \\
\hline A0A034VSG4 & Maternal protein exuperantia & 495 & 51.11 & 26 & $\uparrow 17 \pm 1.02$ & $\uparrow 5.37 \pm 1.74^{\mathrm{a}}$ & $\downarrow 0.27 \pm 0.04$ \\
\hline A0A034WW15 & $40 \mathrm{~S}$ ribosomal protein $\mathrm{S} 3$ & 244 & 80.33 & 26 & $\uparrow 16.58 \pm 0.53$ & $\uparrow 10.31 \pm 1.82$ & $\downarrow 0.54 \pm 0.05$ \\
\hline A0A034VN85 & Eukaryotic translation initiation factor 3 subunit $\mathrm{E}$ & 434 & 38.71 & 15 & $\uparrow 11.54 \pm 0.68$ & $\uparrow 5.3 \pm 2.25^{\mathrm{a}}$ & $\downarrow 0.42 \pm 0.08$ \\
\hline A0A034WLA5 & ATP-dependent RNA helicase vasa, isoform A & 621 & 57.49 & 35 & $\uparrow 11.45 \pm 3.76$ & $\uparrow 6.59 \pm 4.91^{\mathrm{a}}$ & $\downarrow 0.51 \pm 0.04$ \\
\hline A0A034WFM3 & 60S ribosomal protein L3 & 413 & 50.36 & 26 & $\uparrow 10.66 \pm 1.06$ & $\uparrow 5.58 \pm 2.23$ & $\downarrow 0.51 \pm 0.07$ \\
\hline A0A034WMJ8 & $40 \mathrm{~S}$ ribosomal protein $\mathrm{S} 4$ & 280 & 59.29 & 23 & $\uparrow 10.35 \pm 1.61$ & $\uparrow 4.44 \pm 2.48^{\mathrm{a}}$ & $\downarrow 0.41 \pm 0.1$ \\
\hline A0A034VGJ1 & Clustered mitochondria protein & 1362 & 30.54 & 35 & $\uparrow 9.85 \pm 2.74$ & $\uparrow 5.7 \pm 3.47$ & $\downarrow 0.53 \pm 0.02$ \\
\hline A0A034V813 & Acetyl-CoA carboxylase & 2391 & 34.84 & 58 & $\uparrow 9.8 \pm 1.2$ & $\uparrow 5.48 \pm 1.47$ & $\downarrow 0.51 \pm 0.03$ \\
\hline A0A034WGD2 & Ribosomal L1 domain-containing protein & 608 & 32.07 & 18 & $\uparrow 9.37 \pm 0.71$ & $\uparrow 2.86 \pm 1.34$ a & $\downarrow 0.29 \pm 0.06$ \\
\hline A0A034VH20 & Piwi-like protein & 900 & 49.56 & 37 & $\uparrow 8.88 \pm 2.99$ & $\uparrow 3.75 \pm 3.08^{\mathrm{a}}$ & $\downarrow 0.35 \pm 0.05$ \\
\hline A0A034VGU8 & Eukaryotic translation initiation factor 2 subunit 3 & 475 & 45.68 & 16 & $\uparrow 8.09 \pm 1.03$ & $\uparrow 4.25 \pm 1.63$ a & $\downarrow 0.49 \pm 0.05$ \\
\hline A0A034VGS8 & Tubulin $\beta-3$ chain & 454 & 60.79 & 27 & $\uparrow 7.51 \pm 0.22$ & $\uparrow 3.32 \pm 1.16^{\mathrm{a}}$ & $\downarrow 0.42 \pm 0.08$ \\
\hline A0A034V5U3 & Staphylococcal nuclease domain-containing protein 1 & 928 & 64.12 & 47 & $\uparrow 7.34 \pm 0.89$ & $\uparrow 2.91 \pm 1.09$ a & $\downarrow 0.35 \pm 0.04$ \\
\hline A0A034VX01 & DNA-binding protein modulo & 578 & 37.89 & 22 & $\uparrow 7.2 \pm 1.01$ & $\uparrow 2.62 \pm 0.83^{\mathrm{a}}$ & $0.35 \pm 0.01$ \\
\hline A0A034VMS2 & Peptide methionine sulfoxide reductase & 243 & 29.63 & 6 & $\uparrow 6.68 \pm 0.34$ & $\uparrow 3.19 \pm 0.6^{\mathrm{a}}$ & $\downarrow 0.45 \pm 0.04$ \\
\hline A0A034VUY6 & Methenyltetrahydrofolate synthase domain-containing protein & 538 & 56.51 & 32 & $\uparrow 5.91 \pm 0.39$ & $1.09 \pm 0.49^{b}$ & $\downarrow 0.16 \pm 0.03$ \\
\hline A0A034WNF6 & Importin subunit $\alpha$ & 519 & 29.09 & 12 & $\uparrow 5.51 \pm 0.44$ & $1 \pm 0.32^{b}$ & $\downarrow 0.19 \pm 0.02$ \\
\hline A0A034VER3 & Eukaryotic translation initiation factor 3 subunit $C$ & 923 & 37.92 & 32 & $\uparrow 5.49 \pm 0.72$ & $\uparrow 2.35 \pm 1.36^{\mathrm{a}}$ & $\downarrow 0.42 \pm 0.09$ \\
\hline A0A034W2L3 & Citrate synthase & 257 & 63.42 & 15 & $\uparrow 5.37 \pm 0.42$ & $\uparrow 1.75 \pm 1.24^{\mathrm{a}}$ & $\downarrow 0.31 \pm 0.09$ \\
\hline A0A034VH70 & La-related protein & 1206 & 24.71 & 20 & $\uparrow 5.31 \pm 0.28$ & $\uparrow 2.77 \pm 0.4^{\mathrm{a}}$ & $\downarrow 0.5 \pm 0.03$ \\
\hline A0A034VA48 & Prolyl 4-hydroxylase subunit $\alpha 2$ & 561 & 23.53 & 9 & $\uparrow 5.19 \pm 0.6$ & $1.08 \pm 0.28^{\mathrm{b}}$ & $\downarrow 0.21 \pm 0.01$ \\
\hline A0A034W6X9 & GTP-binding protein $128 \mathrm{up}$ & 368 & 52.45 & 16 & $\uparrow 5.12 \pm 0.22$ & $\uparrow 2.39 \pm 0.93$ a & $\downarrow 0.48 \pm 0.08$ \\
\hline A0A034WIR4 & DnaJ-like protein subfamily $\mathrm{C}$ member 2 & 618 & 28.48 & 14 & $\uparrow 4.9 \pm 0.62$ & $\uparrow 2.81 \pm 0.79$ a & $\downarrow$ \\
\hline A0A034WLF6 & FK506-binding protein 59 & 437 & 59.27 & 29 & $\uparrow 4.84 \pm 0.23$ & $\uparrow 2.14 \pm 1.3^{\mathrm{a}}$ & $0.41 \pm 0.11$ \\
\hline A0A034V6Q5 & mRNA turnover protein 4 -like protein & 257 & 28.79 & 8 & $\uparrow 4.61 \pm 0.36$ & $\uparrow 2.13 \pm 0.13^{\mathrm{a}}$ & $\downarrow 0.47 \pm 0.04$ \\
\hline A0A034W8Z3 & Putative ATP-dependent RNA helicase DDX43 & 662 & 47.28 & 27 & $\uparrow 4.6 \pm 0.28$ & $\uparrow 2.5 \pm 0.55^{\mathrm{a}}$ & $\downarrow 0.51 \pm 0.04$ \\
\hline A0A034VYR3 & Nucleolar protein 58 & 728 & 43.82 & 30 & $\uparrow 4.56 \pm 0.83$ & $\uparrow 1.98 \pm 1.14^{\mathrm{a}}$ & $\downarrow 0.41 \pm 0.07$ \\
\hline A0A034WSY7 & Nucleolar GTP-binding protein & 651 & 23.04 & 13 & $\uparrow 3.91 \pm 0.06$ & $1.14 \pm 0.41^{\mathrm{b}}$ & $\downarrow 0.28 \pm 0.05$ \\
\hline A0A034V087 & Guanine nucleotide-binding-like protein 3-like protein & 610 & 29.02 & 16 & $\uparrow 3.67 \pm 0.47$ & $0.76 \pm 0.24 \mathrm{~b}$ & $\downarrow 0.2 \pm 0.01$ \\
\hline A0A034V7N4 & Myosin heavy chain $95 \mathrm{~F}$ & 1240 & 28.63 & 32 & $\uparrow 3.6 \pm 0.57$ & $1.09 \pm 0.56^{b}$ & $\downarrow 0.3 \pm 0.06$ \\
\hline A0A034VCY4 & Tudor domain-containing protein 7 & 956 & 25.63 & 18 & $\uparrow 3.41 \pm 0.76$ & $0.85 \pm 0.49^{b}$ & $\downarrow 0.25 \pm 0.05$ \\
\hline A0A034WCL6 & Peptidyl-prolyl cis-trans isomerase FKBP6 & 478 & 43.93 & 16 & $\uparrow 3.22 \pm 0.32$ & $\uparrow 1.53 \pm 0.39$ a & $\downarrow 0.46 \pm 0.04$ \\
\hline A0A034VFT5 & tRNA (Cytosine(34)-C(5))-methyltransferase & 728 & 33.52 & 16 & $\uparrow 3.05 \pm 0.54$ & $\uparrow 1.78 \pm 0.68^{\mathrm{a}}$ & $\downarrow 0.57 \pm 0.03$ \\
\hline A0A034VFV1 & Cytosolic 10 -formyltetrahydrofolate dehydrogenase & 687 & 47.02 & 23 & $\uparrow 3.04 \pm 0.23$ & $1.42 \pm 0.26^{b}$ & $\downarrow 0.48 \pm 0.04$ \\
\hline $\mathrm{A} 0 \mathrm{~A} 034 \mathrm{VJX} 4$ & Very long-chain fatty-acid-CoA ligase bubblegum & 666 & 30.33 & 15 & $\uparrow 2.95 \pm 0.41$ & $1.3 \pm 0.44^{b}$ & $\downarrow 0.43 \pm 0.04$ \\
\hline
\end{tabular}

a the fold change is $>1.5$ - or $<0.67$-fold but at least one $p$-value $>0.05$; $^{\text {b }}$ no difference of protein abundance; " $\uparrow$ " represents protein abundance up-regulation; " $\downarrow$ " represents protein abundance down-regulation; Proteins with no functional annotation, and also sequence coverage $\leq 20 \%$ were not listed in this table. Ov-1, ov- 6 and ov-9 represent the ovary from 1 -, 6 - and 9-day-old B. dorsalis adult. 
In this study, seven eIFs were identified to be highly abundant in the late stage, indicating their potential roles in ovarian development. Generally, translational repression of mRNAs in the eggs of various insects have been confirmed by interactions, either direct or via intermediate proteins, of repressive factors bound to the $3^{\prime}$-UTRs of proteins in the eIF4E family bound to the $5^{\prime}$-cap of transcripts [38]. In mouse oocyte, a oocyte-specific eIF4E is highly expressed in fully grown oocytes [39]. Insects also rely on the regulated translation of select maternal mRNAs to control oocyte maturation and the initial stages of embryogenesis. These transcripts usually remain silent until their translations are temporally and spatially required during early development. Increasing evidence suggests that eIF4E interacts with cell-specific molecules to control translation during oogenesis and early development in insects [40]. In Drosophila, the Cup protein directly associated with eIF4E is known to be crucial for diverse aspects of female germ-line development [41]. A reduction in eIF4E activity deteriorates the development of ovaries [42]. Only one of these seven eIFs (eIF3C) were transcriptionally validated by qRT-PCR and was shown to have a pattern that differed from protein profiles in the proteomics data.

In the present study, four Hsps were identified at low amounts in newly emerged ovaries, indicating their likely function in oogenesis during ovary maturation in B. dorsalis. A follicle cell specific Hsp83 was identified in mature ovaries of Tribolium castaneum [43]. Moreover, Hsp83 has been reported to function as a component of cap-binding complex and to interact with eIF4E in regulating oogenesis at early stages of egg chamber development during oogenesis in Drosophila [44]. Hsps in the reproductive organs were also reported in testis of $B$. dorsalis, such as Hsp70s and Hsp90s [8]. In addition, a small Hsp (sHsp27) was identified as highly expressed in germline nurse cells throughout oocyte development at the late stages of oogenesis in Drosophila [45]. Stage and cell-specific expression of sHsp27 was recently identified to be differentially expressed and to be located in the ovary during oogenesis of Ceratitis capitata [46]. In B. mori, six sHsps were identified to be highly expressed in the ovary [47]. In this study, one Hsp (Hsp60, I1SWI8) was evaluated at the transcriptional level, which showed increased abundance during ovary development (Figure 7).

The intracellular localization of the $26 \mathrm{~S}$ proteasome in the different ovarian cell types of D. melanogaster has been well-studied. During the pre-vitellogenic phase of oogenesis in Drosophila, cytoplasmic $26 \mathrm{~S}$ proteasome is observed in the nurse cells and follicular epithelial cells. However, a characteristic subcellular redistribution occurs in the ovarian cells during the vitellogenic phase of oogenesis, indicating a strictly tissue- and developmental stage-specific distribution [48]. Here, we identified four $26 \mathrm{~S}$ proteasome non-ATPase regulatory subunits that were highly abundant in the vitellogenic stages. Their high accumulation in the oocyte was also reported in D. melanogaster [49]. However, their mechanism of regulation in the oocyte remains unknown. It was not surprising that Vg was identified as highly abundant in ov- 6 and ov-9 ovaries. In insects, Vg is synthesized in the fat body in a process that involves substantial structural modifications of the nascent protein prior to its secretion and transport to the ovary [50]. It has been reported that Vgs of B. dorsalis are also expressed in the ovary, indicating a complementary $\mathrm{Vg}$ function in ovarian development [51]. In this study, a Vg was identified as abundant in the late ovary, as well as the Vg receptor (S4TMC4).

\subsection{DEPs Highly Abundant in Ovary of 9-Day-Old Adult}

A total of 59 DEPs were highly abundant in the mature ovary (Table 3), among which 17 DEPs were increasingly abundant during the ovarian development. These included defective chorion-1 protein, chorion peroxidase, $\operatorname{Imp} \alpha, \operatorname{Imp} \beta$, nuclear pore complex protein (Nup205), tripeptidyl-peptidase 2, and GST. Other proteins were also identified as highly abundant in mature ovary and include chorion protein S36, myotubularin-related protein, juvenile hormone epoxide hydrolase (JHEH1), and insulin receptor. Most of these DEPs may function in oocyte maturation. Six DEPs showed a similar increasing gene expression pattern along with an increase in the protein levels, while only one DEP (chorion protein s36) out of six DEPs abundant in ov-9 showed a consistent transcriptional expression specifically in ov-9. The remaining five of the tested proteins had increasing expression patterns (Figure 8). 
Table 3. Proteins highly abundant in ovary of 9-day-old adult of Bactrocera dorsalis.

\begin{tabular}{|c|c|c|c|c|c|c|c|}
\hline Protein ID & Annotation & Length & Coverage & Peptides & Ov-6/Ov-1 ${ }^{a, b}$ & Ov-9/Ov-1 & Ov-9/Ov-6 \\
\hline A0A034WSR6 & Defective chorion-1 protein & 723 & 28.49 & 25 & $\uparrow 27.31 \pm 1.25$ & $\uparrow 45.64 \pm 1.03$ & $\uparrow 2.8 \pm 0.2$ \\
\hline A0A034W812 & Importin subunit $\alpha$ & 520 & 63.65 & 22 & $\uparrow 10.77 \pm 0.98$ & $\uparrow 26.6 \pm 1.41$ & $\uparrow 3.62 \pm 0.43$ \\
\hline A0A034VZV8 & Chorion peroxidase & 836 & 40.31 & 29 & $\uparrow 12 \pm 1.27$ & $\uparrow 20.11 \pm 2.27$ & $\uparrow 2.56 \pm 0.39$ \\
\hline A0A034UZ33 & Importin subunit $\beta$ & 885 & 37.51 & 26 & $\uparrow 10.39 \pm 1.97$ & $\uparrow 16.2 \pm 2.62$ & $\uparrow 1.94 \pm 0.02$ \\
\hline A0A034V3F4 & Tripeptidyl-peptidase 2 & 1353 & 47.97 & 54 & $\uparrow 4.9 \pm 0.24$ & $\uparrow 11.08 \pm 0.67$ & $\uparrow 2.61 \pm 0.16$ \\
\hline A0A034V085 & Proteasome-associated protein ECM29-like protein & 1889 & 26.95 & 41 & $\uparrow 7.64 \pm 2.22$ & $\uparrow 11.06 \pm 3.02$ & $\uparrow 1.6 \pm 0.04$ \\
\hline A0A034VPR3 & Elongation factor Tu GTP-binding domain-containing protein 1 & 1043 & 31.06 & 28 & $\uparrow 7.36 \pm 0.75$ & $\uparrow 10.71 \pm 1$ & $\uparrow 1.62 \pm 0.04$ \\
\hline A0A034WID4 & Putative cation-transporting ATPase 13A1 & 1216 & 18.67 & 19 & $\uparrow 6.44 \pm 0.93$ & $\uparrow 10.57 \pm 1.35$ & $\uparrow 1.79 \pm 0.07$ \\
\hline A0A034VC08 & Xaa-Pro dipeptidase & 480 & 42.29 & 18 & $\uparrow 5.88 \pm 0.25$ & $\uparrow 10.51 \pm 0.34$ & $\uparrow 2.09 \pm 0.08$ \\
\hline A0A034VG52 & Adenosylhomocysteinase & 509 & 53.24 & 23 & $\uparrow 6.14 \pm 0.98$ & $\uparrow 9.11 \pm 1.26$ & $\uparrow 1.66 \pm 0.04$ \\
\hline A0A034VV21 & Nuclear pore complex protein Nup205 & 1088 & 22.79 & 20 & $\uparrow 4.06 \pm 0.49$ & $\uparrow 7.84 \pm 0.87$ & $\uparrow 2.11 \pm 0.04$ \\
\hline A0A034VMD8 & Cytoskeleton-associated protein 5 & 1227 & 26.57 & 26 & $\uparrow 3.15 \pm 0.56$ & $\uparrow 6.72 \pm 1.11$ & $\uparrow 2.26 \pm 0.11$ \\
\hline A0A034WDU4 & Endoplasmin & 797 & 56.21 & 46 & $\uparrow 3.9 \pm 0.12$ & $\uparrow 6.47 \pm 0.19$ & $\uparrow 1.74 \pm 0.03$ \\
\hline A0A034W4A7 & Glutathione S-transferase 1-1 & 207 & 49.76 & 11 & $\uparrow 2.74 \pm 0.06$ & $\uparrow 5.86 \pm 0.51$ & $\uparrow 2.26 \pm 0.2$ \\
\hline A0A034WP50 & Chorion protein S36 & 233 & 54.08 & 10 & $\uparrow 8.94 \pm 1.36^{\mathrm{a}}$ & $\uparrow 36.76 \pm 2.19$ & $\uparrow 15.69 \pm 1.6$ \\
\hline A0A034VUE5 & Myotubularin-related protein 14 & 765 & 30.59 & 18 & $\uparrow 8.44 \pm 0.67^{\mathrm{a}}$ & $\uparrow 29.22 \pm 1.46$ & $\uparrow 6.21 \pm 0.44$ \\
\hline A0A034W787 & Juvenile hormone epoxide hydrolase 1 & 459 & 32.46 & 18 & $\uparrow 16.8 \pm 1.38^{\mathrm{a}}$ & $\uparrow 26.88 \pm 1.96$ & $\uparrow 2.64 \pm 0.29$ \\
\hline A0A034VVU8 & Muskelin & 890 & 38.2 & 32 & $\uparrow 6.51 \pm 0.55^{\mathrm{a}}$ & $\uparrow 17.65 \pm 3.35$ & $\uparrow 4.04 \pm 0.9$ \\
\hline A0A034W030 & MAP kinase-activating death domain protein & 2175 & 7.724 & 13 & $\uparrow 5.65 \pm 0.65^{\mathrm{a}}$ & $\uparrow 17.31 \pm 1.03$ & $\uparrow 3.84 \pm 0.3$ \\
\hline A0A034W1D2 & UDP-glucuronosyltransferase $1-1$ & 640 & 8.594 & 5 & $\uparrow 4.31 \pm 0.54^{\mathrm{a}}$ & $\uparrow 12.79 \pm 1.88$ & $\uparrow 3.44 \pm 0.53$ \\
\hline A0A034W397 & Dual specificity mitogen-activated protein kinase kinase dSOR1 & 397 & 44.58 & 18 & $\uparrow 5.39 \pm 0.1^{\mathrm{a}}$ & $\uparrow 11.14 \pm 0.87$ & $\uparrow 2.67 \pm 0.14$ \\
\hline A0A034WCE7 & Insulin receptor & 963 & 30.43 & 21 & $\uparrow 3.01 \pm 0.76^{\mathrm{a}}$ & $\uparrow 10.2 \pm 1.62$ & $\uparrow 4.24 \pm 0.39$ \\
\hline A0A034W9D5 & Glutaminyl-peptide cyclotransferase-like protein & 330 & 15.45 & 5 & $\uparrow 2.57 \pm 0.71^{\mathrm{a}}$ & $\uparrow 9.6 \pm 1.15$ & $\uparrow 4.79 \pm 1$ \\
\hline A0A034WBX7 & Ribonucleoside-diphosphate reductase & 804 & 46.27 & 34 & $\uparrow 2.49 \pm 0.45^{\mathrm{a}}$ & $\uparrow 9.34 \pm 1.45$ & $\uparrow 3.83 \pm 0.28$ \\
\hline A0A034V8K5 & Aminopeptidase $\mathrm{N}$ & 1035 & 19.81 & 16 & $\uparrow 2.64 \pm 0.68^{a}$ & $\uparrow 7.97 \pm 1.66$ & $\uparrow 3.55 \pm 0.26$ \\
\hline A0A034VKU6 & Myotubularin-related protein 3 & 1279 & 17.98 & 18 & $\uparrow 1.86 \pm 0.21^{\mathrm{a}}$ & $\uparrow 7.79 \pm 0.76$ & $\uparrow 4.51 \pm 0.12$ \\
\hline A0A034VIB7 & Protein phosphatase 1B & 370 & 40.54 & 13 & $\uparrow 3 \pm 0.31^{\mathrm{a}}$ & $\uparrow 7.73 \pm 0.75$ & $\uparrow 2.73 \pm 0.23$ \\
\hline A0A034WC12 & Cytosolic endo- $\beta-N$-acetylglucosaminidase & 604 & 30.3 & 15 & $\uparrow 2.21 \pm 0.39^{\mathrm{a}}$ & $\uparrow 7.67 \pm 1.22$ & $\uparrow 3.88 \pm 0.72$ \\
\hline A0A034WUY1 & Ubiquitin carboxyl-terminal hydrolase & 227 & 74.89 & 11 & $\uparrow 4.46 \pm 0.45^{\mathrm{a}}$ & $\uparrow 7.42 \pm 0.68$ & $\uparrow 1.9 \pm 0.07$ \\
\hline A0A034VK02 & Dihydrolipoyl dehydrogenase & 504 & 58.33 & 26 & $\uparrow 3.79 \pm 0.22^{\mathrm{a}}$ & $\uparrow 6.88 \pm 0.35$ & $\uparrow 1.98 \pm 0.15$ \\
\hline A0A034VAA7 & Centrosomin & 992 & 10.58 & 8 & $\uparrow 1.57 \pm 0.21^{\mathrm{a}}$ & $\uparrow 6.77 \pm 0.68$ & $\uparrow 4.8 \pm 0.35$ \\
\hline A0A034W647 & Peptidyl-prolyl cis-trans isomerase D & 442 & 34.62 & 15 & $\uparrow 2.29 \pm 0.07^{\mathrm{a}}$ & $\uparrow 6.55 \pm 0.29$ & $\uparrow 2.98 \pm 0.2$ \\
\hline G9F9Y5 & Chitinase & 483 & 24.43 & 8 & $1.18 \pm 0.17^{\mathrm{b}}$ & $\uparrow 6.26 \pm 0.61$ & $\uparrow 5.86 \pm 0.67$ \\
\hline A0A034WDM9 & $26 \mathrm{~S}$ protease regulatory subunit $6 \mathrm{~A}$ & 428 & 63.55 & 25 & $\uparrow 3.65 \pm 0.06^{\mathrm{a}}$ & $\uparrow 5.63 \pm 0.08$ & $\uparrow 1.68 \pm 0.06$ \\
\hline
\end{tabular}


Table 3. Cont.

\begin{tabular}{|c|c|c|c|c|c|c|c|}
\hline Protein ID & Annotation & Length & Coverage & Peptides & Ov-6/Ov-1 ${ }^{a, b}$ & Ov-9/Ov-1 & Ov-9/Ov-6 \\
\hline A0A034WB53 & Cullin-5 & 850 & 16.24 & 12 & $0.95 \pm 0.23^{b}$ & $\uparrow 5.13 \pm 0.53$ & $\uparrow 5.75 \pm 1.07$ \\
\hline A0A034W865 & Gamma-tubulin complex component 3-like protein & 951 & 19.77 & 15 & $\uparrow 1.86 \pm 0.13^{\mathrm{a}}$ & $\uparrow 4.98 \pm 0.65$ & $\uparrow 2.88 \pm 0.36$ \\
\hline A0A034WNA4 & GMP synthase (Glutamine-hydrolyzing) & 683 & 53.44 & 30 & $\uparrow 1.94 \pm 0.1^{\mathrm{a}}$ & $\uparrow 4.92 \pm 0.18$ & $\uparrow 2.68 \pm 0.09$ \\
\hline A0A034W2K6 & Major royal jelly protein 1 & 425 & 40.24 & 14 & $0.67 \pm 0.05^{b}$ & $\uparrow 4.68 \pm 0.62$ & $\uparrow 6.5 \pm 0.67$ \\
\hline A0A034WHL8 & Venom carboxylesterase- 6 & 549 & 25.14 & 12 & $1.31 \pm 0.07^{b}$ & $\uparrow 4.68 \pm 0.27$ & $\uparrow 3.84 \pm 0.3$ \\
\hline A0A034WMG0 & Ubiquitin carboxyl-terminal hydrolase & 1110 & 31.08 & 29 & $\uparrow 2.24 \pm 0.47^{\mathrm{a}}$ & $\uparrow 4.28 \pm 0.91$ & $\uparrow 2.08 \pm 0.18$ \\
\hline A0A034VRJ4 & Neutral $\alpha$-glucosidase $\mathrm{AB}$ & 449 & 45.88 & 18 & $1.1 \pm 0.18^{\mathrm{b}}$ & $\uparrow 3.91 \pm 0.57$ & $\uparrow 3.5 \pm 0.24$ \\
\hline A0A034VK16 & Cytoskeleton-associated protein 5 & 734 & 32.7 & 20 & $\uparrow 2.41 \pm 0.18^{\mathrm{a}}$ & $\uparrow 3.9 \pm 0.22$ & $\uparrow 1.69 \pm 0.06$ \\
\hline A0A034W7H6 & Cyclin-dependent kinase 5 -like protein & 293 & 14.68 & 4 & $1.22 \pm 0.17^{\mathrm{b}}$ & $\uparrow 3.82 \pm 0.33$ & $\uparrow 3.46 \pm 0.48$ \\
\hline A0A034V2C6 & Serine/threonine-protein kinase Warts & 610 & 16.23 & 7 & $1.41 \pm 0.06^{\mathrm{b}}$ & $\uparrow 3.62 \pm 0.07$ & $\uparrow 2.66 \pm 0.14$ \\
\hline A0A034W7N0 & Protein NASP-like protein & 427 & 41.22 & 15 & $\uparrow 1.67 \pm 0.1^{\mathrm{a}}$ & $\uparrow 3.4 \pm 0.19$ & $\uparrow 2.11 \pm 0.12$ \\
\hline A0A034WQ10 & Cullin- $4 \mathrm{~A}$ & 841 & 27.59 & 20 & $\uparrow 1.79 \pm 0.14^{\mathrm{a}}$ & $\uparrow 3.35 \pm 0.17$ & $\uparrow 1.92 \pm 0.12$ \\
\hline A0A034VQ97 & CD109 antigen & 1430 & 30.49 & 39 & $0.87 \pm 0.14^{b}$ & $\uparrow 3.11 \pm 0.21$ & $\uparrow 3.78 \pm 0.54$ \\
\hline A0A034VKF8 & Cysteine and histidine-rich protein 1-like protein & 447 & 30.2 & 10 & $1.26 \pm 0.03^{b}$ & $\uparrow 2.88 \pm 0.13$ & $\uparrow 2.34 \pm 0.12$ \\
\hline A0A034WBQ5 & $\alpha-1,4$ glucan phosphorylase & 845 & 61.07 & 50 & $1.33 \pm 0.11^{b}$ & $\uparrow 2.84 \pm 0.33$ & $\uparrow 2.2 \pm 0.3$ \\
\hline A0A034VJU9 & 85/88 kDa calcium-independent phospholipase A2 & 872 & 23.28 & 19 & $1.36 \pm 0.11^{b}$ & $\uparrow 2.8 \pm 0.08$ & $\uparrow 2.17 \pm 0.2$ \\
\hline A0A034WAP1 & $\beta$-ureidopropionase & 385 & 41.3 & 11 & $0.8 \pm 0.03^{b}$ & $\uparrow 1.98 \pm 0.09$ & $\uparrow 2.41 \pm 0.12$ \\
\hline
\end{tabular}

${ }^{a}$ the fold change is $\geq 1.5$ - or $\leq 0.67$-fold but at least one $p$-value $\geq 0.05$; $^{\mathrm{b}}$ no difference of protein abundance; " $\uparrow$ " represents protein abundance up-regulation; " $\downarrow$ " represents protein abundance down-regulation; Proteins with no functional annotation, and also sequence coverage $\leq 20 \%$ were not listed in this table. Ov-1, ov- 6 and ov- 9 represent the ovary from 1-, 6 - and 9-day-old B. dorsalis adult. 

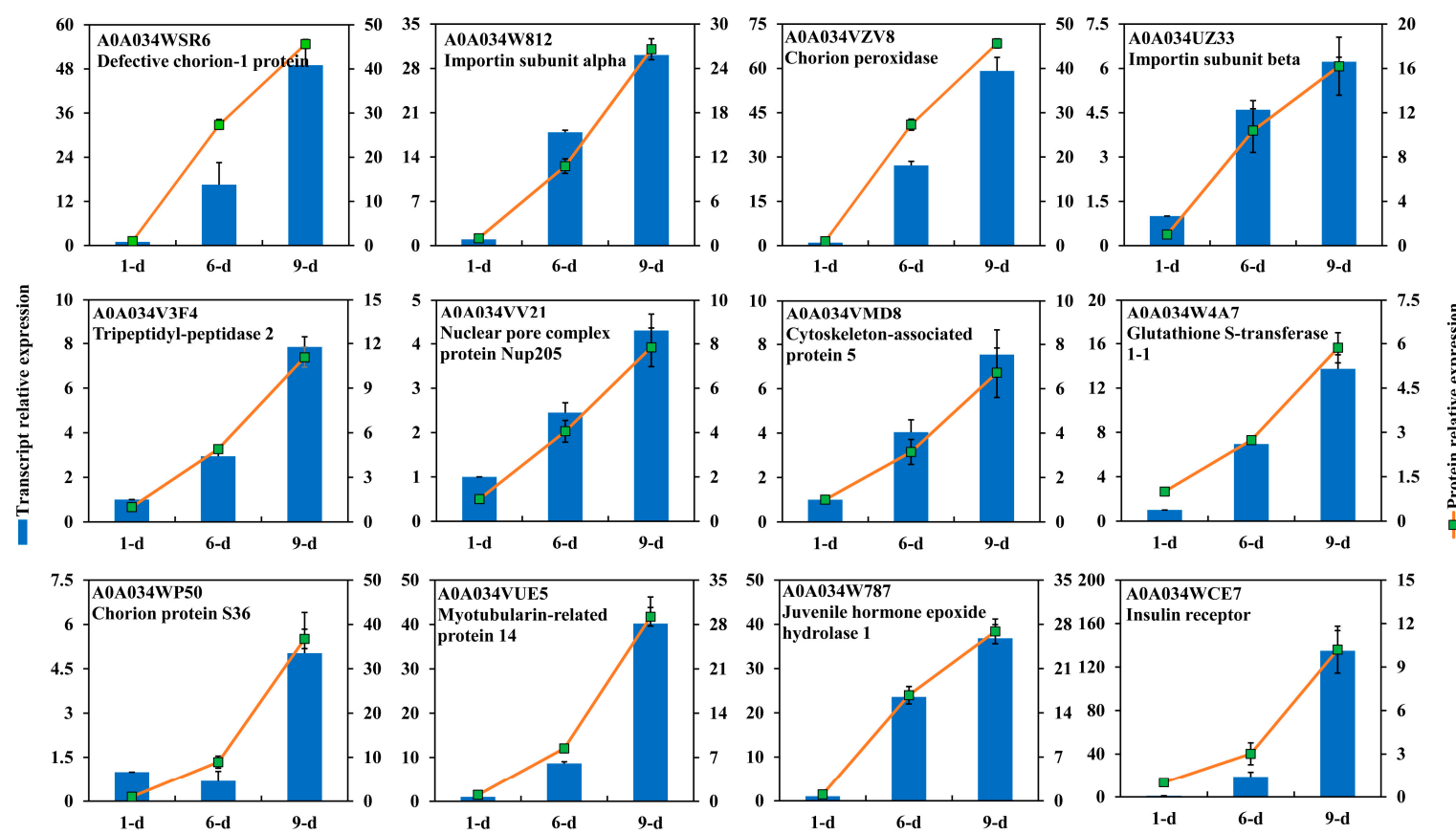

Figure 8. Transcriptional expression of proteins highly abundant in ovary of 9-day-old Bactrocera dorsalis adult. Gene expression was calculated as Figure 6.

$\operatorname{Imp} \beta$ was originally described to participate in the import of proteins that carry a classical nuclear localization signal (NLS) into the nucleus, a key player in nuclear protein import [52]. Nurse cells synthesize and transfer Imp $\beta$ into the oocyte cytoplasm from late stage of oogenesis in D. melanogaster, and $\operatorname{Imp} \beta$ gene appears to be ubiquitously expressed in embryonic cells [53]. $\operatorname{Imp} \beta$ interacts with the nuclear pore complex (NPC), NLS dependent protein and also Imp $\alpha$ forming a trimeric complex. Thus, Imp $\alpha$ plays a role in oogenesis in association with $\operatorname{Imp} \beta$. It has been demonstrated that the concentration of Imp $\alpha 3$ within the nurse cell nuclei increases during stages 7-10 (vitellogenic stage) of oogenesis, and plays a crucial role in the completion of oogenesis [54]. Imp $\alpha 2$ protein is uniformly distributed in the nurse cell cytoplasm with a moderate accumulation along the oocyte cortex [55]. Indeed, there are three $\operatorname{Imp} \alpha$ with a specific and limited expression profiles in D. melanogaster spermatogenesis, but they play different roles in oogenesis [56]. Homologous Imp $\alpha$ and $\operatorname{Imp} \beta$ were both identified as the most abundant in the mature ovaries of $B$. dorsalis. Consistent transcriptional expression indicated their crucial roles in oocyte maturation. The Imp $\alpha$ defect in female Drosophila causes sterility and leads to the arrest of oogenesis in late stage $[54,56,57]$. The roles of Imps in the development of both larval and adult tissues were also uncovered, suggesting a potential for their use in pest control. However, their functions and mechanisms of regulation during oogenesis should be well addressed in B. dorsalis. In addition, four NPCs were also identified as abundant in ov-6 while another NPC was more abundant in ov-9. Besides, we also identified three $\operatorname{Imp}(\operatorname{Imp} 4,-5$, -7) that were highly expressed during the vitellogenic development. Imp7 is distantly related to the proteins of $\operatorname{Imp} \beta$ family, and is required for the proper formation of muscle-tendon adhesion sites in developing Drosophila embryos [58]. The high expressions of Imp4 and Imp5 were also determined by qRT-PCR, indicating their important roles in oocyte development (Figure 7).

The eggshell, including Vmps and chorion proteins, is a specialized extracellular matrix that is synthesized between the oocyte and overlaying somatic follicle cells during the late stages of oogenesis. As follicles mature, they move through the ovarioles and undergo successively vitellogenesis and choriogenesis (eggshell formation). In insects such as Drosophila and Bombyx, Vmp is synthesized by and secreted from the cells of the follicular epithelium [59]. In Drosophila, eggshell constituents are synthesized in the follicle cells at the beginning of the vitellogenic stage (stage 8 ) in a well-defined 
spatial and temporal pattern reflecting their contribution to the eggshell. Vmp gene is expressed earlier than chorion gene, which begins at late stage (stage 11) and proceeds until the end of oogenesis [60]. In B. mori, mutation of Vmp is investigated in the egg-lethal phenotype [61]. Another Vmp (Vmp90) is identified to play an essential role in the developing ovarian follicle [62]. In the present study, Vmp26Aa identified in ov-6 was found to be considerably abundant until the mature stage. This protein also showed higher transcriptional expression in ov-6 (Figure 7). Similar to Drosophila, high abundance of chorion and chorion peroxidase were also investigated in mature ovary at both transcriptional and protein levels (Figure 8). In addition to the component of eggshell, an ovarian follicular epithelium protein was identified to be expressed exclusively in the cells during specific stages of vitellogenesis and functionally associated with vitelline membrane that contributes to the structural integrity of the follicle [63]. Chorion is produced during the late stages of oogenesis by epithelial follicle cells and develops into a highly organized multi-layered structure that exhibits regional specialization and radial complexity. In this study, a chorion s36 was identified as highly abundant in the mature ovary. In Drosophila, a homologous protein plays a crucial role in regulating the morphogenetic integrity of dorsal appendages in follicles inducing severe structural irregularities on chorion's surface and entirely impairing fly's fertility [64]. Moreover, a chorion peroxidase was also found to be abundant in mature ovary. This specific peroxidase is also identified in D. melanogaster and B. oleae, and is reported to be involved in the chorion hardening process through protein crosslinking mediated by the formation of di- and tri-tyrosine bonds $[65,66]$.

Ovarian development is triggered by the steroid hormone, 20-hydroxy-ecdysone, which plays key role in Drosophila oogenesis, as its activity is required at multiple steps during egg chamber maturation [67]. Recently, its involvement has been reported on eggshell production by controlling chorion gene transcription and amplification [68]. A Halloween gene cytochrome, P450 306a1 (Phantom), was identified abundant in the vitellogenic stage of oogenesis in B. dorsalis. Transcriptional data also showed its high expression. A JHEH1 was also found to be highly expressed in the vitellogenic stage in this study, revealing a complementary regulation during ovarian development. Similarly, insulin receptor was found to be abundant in mature ovary. It was previously reported that insulin plays a role in ovarian development in B. dorsalis [69]. It is well known that in Blattella germanica, insulin receptor regulates juvenile hormone biosynthesis and vitellogenin production through nutritional signals [70]. In addition, regulation of notch signal pathway in follicle formation was investigated in mouse ovary [71]. Proteins involved in these pathways were also identified to be abundant in the ovary development of $B$. dorsalis.

\section{Materials and Methods}

\subsection{Insects Culture}

The stock flies were originally collected from Hainan Province of China in 2008. All insects were maintained at $27.5 \pm 0.5{ }^{\circ} \mathrm{C}, 75 \pm 5 \%$ relative humidity, and a $14: 10 \mathrm{~h}$ (light: dark) photoperiod as described previously [4]. Under these conditions, the flies grew well and adults reached sexual maturity at 8-9 days after emergency [2]. Newly emerged female adults were separated immediately and ovaries were dissected from 1-, 3-, 5-, 7-, 9-day-old virgin females for image capture (Leica Microsystems, Wetzlar, Germany). Because of the differences in ovarian development, only ovaries of 6-day-old adults that appeared similar to the graphics between Figure 1C to Figure 1D were collected. All samples were stored as $-80^{\circ} \mathrm{C}$ before isolating the proteins. Two biological replicates were prepared for each sample.

\subsection{Protein Extraction}

All samples were powdered in liquid nitrogen and the powder was dissolved in $200 \mu \mathrm{L}$ lysis buffer $(\mathrm{pH}=8.3)$ containing $7 \mathrm{M}$ urea, $2 \mathrm{M}$ thiourea, and $20 \mathrm{mM}$ Tris. Then, $800 \mu \mathrm{L}$ of cold acetone containing $10 \mathrm{mM}$ DTT was added and the mixture was incubated for $2 \mathrm{~h}$ and centrifuged at 13,000 rpm for $20 \mathrm{~min}$ at $15^{\circ} \mathrm{C}$, and the supernatants were discarded. Then, $800 \mu \mathrm{L}$ of cold acetone containing 
$10 \mathrm{mM}$ DTT was added and the mixture was incubated at $56{ }^{\circ} \mathrm{C}$ for $1 \mathrm{~h}$ to break the disulfide bonds in proteins followed by centrifugation as above. The precipitate was dissolved in $100 \mu \mathrm{L}$ of lysis buffer and the protein concentration in the solute was determined by using the Bradford method with bovine serum albumin as a standard [72].

\subsection{Sample Digestion and Labeling}

The extracted protein $(100 \mu \mathrm{g})$ was first diluted in $100 \mu \mathrm{L}$ dissolution buffer. Then, $2 \mu \mathrm{g}$ of trypsin (Promega, Madison, WI, USA) was added and the mixture was diluted with $500 \mu \mathrm{L} \mathrm{NH}_{4} \mathrm{HCO}_{3}$ $(50 \mathrm{mM})$ and incubated for $16 \mathrm{~h}$ at $37{ }^{\circ} \mathrm{C}$ for protein digestion. After protein digestion, equal volume of $0.1 \%$ formic acid was added for acidulation. Peptides were purified on Strata $-X$ C18 pillar, which was activated with methanol and then balanced with $1 \mathrm{~mL}$ of $0.1 \%$ formic acid three times. Then, the peptides were washed with $0.1 \%$ formic acid $+5 \%$ acetonitrile twice and eluted with $1 \mathrm{~mL}$ of $0.1 \%$ formic acid $+80 \%$ acetonitrile. Eluted peptides were vacuum dried (ThermoFisher Scientific, Asheville, NC, USA) and the dried peptides powder was dissolved in $20 \mu \mathrm{L}$ of $0.5 \mathrm{M}$ tetraethylammonium bromide (TEAB) for peptide labeling. Samples were labeled with the iTRAQ Reagent-8 plex Multiplex Kit (AB Sciex, Framingham, MA, USA) according to the manufacturer's instructions. Six samples were labeled with different iTRAQ tags: 113 and 114 for ov-1, 115 and 116 for ov-6, and 117 and 118 for ov-9. Equal amounts of the labeled samples were pooled together and then fractionated using high-performance liquid chromatography (HPLC) (Thermo Scientific DINOEX Ultimate 3000 BioRS, Waltham, MA, USA) using a Durashell C18 $(5 \mu \mathrm{m}, 100 \AA, 4.6 \times 250 \mathrm{~mm})$. Finally, 12 fractions were collected.

\subsection{LC-MS/MS Analysis}

LC-MS/MS analysis was performed on an AB SCIEX nanoLC-MS/MS system (Triple TOF 5600 plus, Framingham, MA, USA). Samples were chromatographed on a 120-min gradient from 2-35\% (buffer A $0.1 \%$ formic acid, 5\% acetonitrile, buffer B $0.1 \%$ formic acid, $95 \%$ acetonitrile) after direct injection onto a $20 \mathrm{~cm}$ PicoFrit emitter (New Objective) packed to $20 \mathrm{~cm}$ with Magic C18 with an inner diameter of $3 \mu \mathrm{m}$ at $200 \AA$ stationary phase. MS1 spectra were collected in the range of 360-1460 m/z for $250 \mathrm{~ms}$. The 20 most intense precursors with charge state 2-5 were selected for fragmentation, and MS2 spectra were collected in the range of 50-2000 m/z for $100 \mathrm{~ms}$; precursor ions were excluded from reselection for $15 \mathrm{~s}$.

\subsection{Protein Identification and Functional Annotation}

The original MS/MS file data were submitted to the ProteinPilot Software (AB SCIEX, version 4.0) for data analysis. For protein identification, the Paragon algorithm, which was integrated into ProteinPilot was employed against uniprot database for database searching [73]. Cysteine was modified with iodoacetamide; biological modifications were selected as ID focus, trypsin digestion, the Quantitate, Bias Correction, and Background Correction was checked for protein quantification and normalization. Only proteins with more than one peptides and unused value $\geq 1.3$ were considered for identification.

To determine the biological and functional properties, all the identified proteins were mapped with Gene Ontology Terms (available online: http://geneontology.org/). For this, homology search was first performed for all the identified proteins with a localized NCBI BlastP program against the UniProt database of $B$. dorsalis species. The e-value was set to less than $10^{-5}$, and the best hit for each query sequence was taken into account for GO term matching, which was performed with blast2go v4.5 pipeline [74]. Clusters of Orthologous Groups of Proteins System (COG, available online: http:/ /www. ncbi.nlm.nih.gov/COG/) was employed for the functional annotation of genes from new genomes and for research on genome evolution. To identify candidate biomarkers, we employed hypergeometric test to perform GO enrichment and Pathway enrichment. The GO and COG assignment were also performed within the DEPs in this study. 


\subsection{Protein Quantitative Analysis}

DEPs were determined based on the ratios of differently labeled proteins and $P$ values provided by Proteinpilot. Fold change was calculated as the average comparison pairs among biological replicates. We set restrictive conditions to find the potential proteins involved in the ovarian development in this study. Only the proteins with expression fold changes $\geq 1.5$ - or $\leq 0.67$-fold between all the comparisons of biological replicates, as well as $P$ value of all the differences between protein abundance comparisons $<0.05$ were identified as DEPs. All the DEPs between each comparison were functionally annotated as above. In order to screen for potential target proteins involved in the pre-vitellogenic and vitellogenic stages, proteins highly abundant in each independent stage were thereafter functionally classified.

\subsection{RNA Extraction and RT-qPCR Analysis}

To examine the transcriptional expression of potential functional proteins with high expression in each staged ovary, total RNA from ov-1, ov-6, and ov-9 with the similar morphological ovaries were isolated using TRIZoL reagent (Invitrogen, Carlsbad, CA, USA) following the manufacturer's instructions. First-strand cDNA was obtained from DNase I-treated RNA $(\sim 1 \mu \mathrm{g})$ using a PrimeScript 1st Strand cDNA Synthesis Kit (Takara, Dalian, China). Some DEPs highly expressed in ov-1, ov-6, and ov-9 were randomly selected for qRT-PCR analysis. PCR primers for each DEP were designed using an online tool, Primer 3, based on the corresponding nucleotide sequences in NCBI. For control purposes, a fragment of $\alpha$-tubulin open reading frame was also amplified using gene specific primers [75]. All PCR primers used in the research presented here were list in Table S5. Each reaction consisted of a $10 \mu \mathrm{L}$ volume containing $0.5 \mu \mathrm{L}$ of cDNA template, $5 \mu \mathrm{L}$ of GoTaq qPCR Master Mix (Promega, Madison, WI, USA), $0.5 \mu \mathrm{L}$ of each primer $(10 \mu \mathrm{M})$ and $3.5 \mu \mathrm{L}$ of nuclease-free water. The reaction conditions and calculation of gene expression were essentially as described before [9].

\section{Conclusions}

In conclusion, a total of 4838 proteins were identified in B. dorsalis ovaries. Among these, 612 and 196 proteins were differentially expressed in vitellogenic and mature ovaries, respectively. Together, we identified 153, 44, and 59 proteins to be highly abundant in 1-, 6- and 9-day-old ovaries, respectively. Many DEPs were transcriptionally validated and showed consistent profiles at both transcriptional and translational levels. Many potential target proteins that were highly expressed in the three ovarian stages, including tropomyosin, vitellogenin, eukaryotic translation initiation factor, importin protein, vitelline membrane protein, and chorion protein. Some hormone and signal pathway related proteins were also identified during ovarian development, such as piRNA, notch, insulin, juvenile and ecdysone hormone signal pathways. This is the first report of a global proteome in a tephritid ovary and contributes to our understanding of the complicated processes of ovarian development in insects. These results will also aid in the identification of novel target proteins for improving strategies to control tephritid fruit flies.

Supplementary Materials: Supplementary materials can be found at www.mdpi.com/1422-0067/18/7/1379/s1.

Acknowledgments: We thank Qi-Ya Huang and Hong-Ting Jia for their help of sequence analysis and primer design. This study was partly supported by the National Natural Science Foundation of China (31601640), the Project Funded by China Postdoctoral Science Foundation (2016T90828, 2015M582501), the earmarked fund for the Modern Agro-industry (Citrus) Technology Research System (CARS-27), and the Foundation Project of Southwest University (SWU114049).

Author Contributions: Dong Wei, Jin-Jun Wang and Guy Smagghe conceived and designed the experiments; Dong Wei, Ran Li and Meng-Yi Zhang performed the experiments; Dong Wei, Yu-Wei Liu and Zheng Zhang analyzed the data; Dong Wei and Jin-Jun Wang wrote the paper.

Conflicts of Interest: The authors declare no conflict of interest. The founding sponsors had no role in the design of the study; in the collection, analyses, or interpretation of data; in the writing of the manuscript, and in the decision to publish the results. 


\section{References}

1. Shelly, T.E.; Edu, J.; Pahio, E. Influence of diet and methyl eugenol on the mating success of males of the oriental fruit fly, Bactrocera dorsalis (Diptera: Tephritidae). Fla. Entomol. 2005, 88, 307-313. [CrossRef]

2. Wei, D.; Feng, Y.C.; Wei, D.D.; Yuan, G.R.; Dou, W.; Wang, J.J. Female remating inhibition and fitness of Bactrocera dorsalis (Diptera: Tephritidae) associated with male accessory glands. Fla. Entomol. 2015, 98, 52-58. [CrossRef]

3. Jin, T.; Zeng, L.; Lin, Y.Y.; Lu, Y.Y.; Liang, G.W. Insecticide resistance of the oriental fruit fly, Bactrocera dorsalis (Hendel) (Diptera: Tephritidae), in mainland China. Pest Manag. Sci. 2011, 67, 370-376. [CrossRef] [PubMed]

4. Wang, J.J.; Wei, D.; Dou, W.; Hu, F.; Liu, W.F.; Wang, J.J. Toxicities and synergistic effects of several insecticides against the oriental fruit fly (Diptera: Tephritidae). J. Econ. Entomol. 2013, 106, 970-978. [CrossRef] [PubMed]

5. Chen, L.J.; Meng, Q.Q.; Li, Z.Q.; Zhang, S.Q.; Zeng, L.; Lu, Y.Y. The resistance monitoring of Bactrocera dorsalis in the field of Shenzhen. Chin. Plant Prot. 2015, 35, 63-66.

6. Zheng, W.W.; Peng, T.; He, W.; Zhang, H.Y. High-throughput sequencing to reveal genes involved in reproduction and development in Bactrocera dorsalis (Diptera: Tephritidae). PLoS ONE 2012, 7, e36463. [CrossRef] [PubMed]

7. Geib, S.M.; Calla, B.; Hall, B.; Hou, S.; Manoukis, N.C. Characterizing the developmental transcriptome of the oriental fruit fly, Bactrocera dorsalis (Diptera: Tephritidae) through comparative genomic analysis with Drosophila melanogaster utilizing modENCODE datasets. BMC Genom. 2014, 15, 942. [CrossRef] [PubMed]

8. Wei, D.; Li, H.M.; Yang, W.J.; Wei, D.D.; Dou, W.; Huang, Y.; Wang, J.J. Transcriptome profiling of the testis reveals genes involved in spermatogenesis and marker discovery in the oriental fruit fly, Bactrocera dorsalis. Insect Mol. Biol. 2015, 24, 41-57. [CrossRef] [PubMed]

9. Wei, D.; Tian, C.B.; Liu, S.H.; Wang, T.; Smagghe, G.; Jia, F.X.; Dou, W.; Wang, J.J. Transcriptome analysis to identify genes for peptides and proteins involved in immunity and reproduction from male accessory glands and ejaculatory duct of Bactrocera dorsalis. Peptides 2016, 80, 48-60. [CrossRef] [PubMed]

10. Takemori, N.; Yamamoto, M.T. Proteome mapping of the Drosophila melanogaster male reproductive system. Proteomics 2009, 9, 2484-2493. [CrossRef] [PubMed]

11. Velentzas, A.D.; Anagnostopoulos, A.K.; Velentzas, P.D.; Mpakou, V.E.; Sagioglou, N.E.; Tsioka, M.M.; Katarachia, S.; Manta, A.K.; Konstantakou, E.G.; Papassideri, I.S.; et al. Global proteomic profiling of Drosophila ovary: A high-resolution, unbiased, accurate and multifaceted analysis. Cancer Genom. Proteom. 2015, 12, 369-384.

12. Lin, H. The stem-cell niche theory: Lessons from flies. Nat. Rev. Genet. 2002, 3, 931-940. [CrossRef] [PubMed]

13. Horne-Badovinac, S.; Bilder, D. Mass transit: Epithelial morphogenesis in the Drosophila egg chamber. Dev. Dynam. 2005, 232, 559-574. [CrossRef] [PubMed]

14. Dobens, L.L.; Raftery, L.A. Integration of epithelial patterning and morphogenesis in Drosophila ovarian follicle cells. Dev. Dynam. 2000, 218, 80-93. [CrossRef]

15. Hook, J.; Lemckert, F.; Qin, H.; Schevzov, G.; Gunning, P. Gamma tropomyosin gene products are required for embryonic development. Mol. Cell. Biol. 2004, 24, 2318-2323. [CrossRef] [PubMed]

16. Jang, W.I.; Jo, Y.J.; Kim, H.C.; Jia, J.L.; Namgoong, S.; Kim, N.H. Non-muscle tropomyosin (Tpm3) is crucial for asymmetric cell division and maintenance of cortical integrity in mouse oocytes. Cell Cycle 2014, 13, 2359-2369. [CrossRef] [PubMed]

17. Clayton, L.; Johnson, M.H. Tropomyosin in preimplantation mouse development: Identification, expression, and organization during cell division and polarization. Exp. Cell Res. 1998, 238, 450-464. [CrossRef] [PubMed]

18. Erdelyi, M.; Michon, A.M.; Guichet, A.; Glotzer, J.B.; Ephrussi, A. Requirement for Drosophila cytoplasmic tropomyosin in oskar mRNA localization. Nature 1995, 377, 524-527. [CrossRef] [PubMed]

19. Tetzlaff, M.T.; Jackle, H.; Pankratz, M.J. Lack of Drosophila cytoskeletal tropomyosin affects head morphogenesis and the accumulation of oskar mRNA required for germ cell formation. EMBO J. 1996, 15, 1247-1254. [PubMed]

20. Young, P.E.; Pesacreta, T.C.; Kiehart, D.P. Dynamic changes in the distribution of cytoplasmic myosin during Drosophila embryogenesis. Development 1991, 111, 1-14. [PubMed] 
21. Wheatley, S.; Kulkarni, S.; Karess, R. Drosophila nonmuscle myosin II is required for rapid cytoplasmic transport during oogenesis and for axial nuclear migration in early embryos. Development 1995, 121, 1937-1946. [PubMed]

22. Edwards, K.A.; Kiehart, D.P. Drosophila nonmuscle myosin II has multiple essential roles in imaginal disc and egg chamber morphogenesis. Development 1996, 122, 1499-1511. [PubMed]

23. Jordan, P.; Karess, R. Myosin light chain-activating phosphorylation sites are required for oogenesis in Drosophila. J. Cell Biol. 1997, 139, 1805-1819. [CrossRef] [PubMed]

24. Krauss, J.; de Quinto, S.L.; Nüsslein-Volhard, C.; Ephrussi, A. Myosin-V regulates oskar mRNA localization in the Drosophila oocyte. Curr. Biol. 2009, 19, 1058-1063. [CrossRef] [PubMed]

25. Deng, W.; Leaper, K.; Bownes, M. A targeted gene silencing technique shows that Drosophila myosin VI is required for egg chamber and imaginal disc morphogenesis. J. Cell Sci. 1999, 112, 3677-3690. [PubMed]

26. Zhou, X.H.; Ban, L.P.; Iovinella, I.; Zhao, L.J.; Gao, Q.; Felicioli, A.; Sagona, S.; Pieraccini, G.; Pelosi, P.; Zhang, L. Diversity, abundance, and sex-specific expression of chemosensory proteins in the reproductive organs of the locust Locusta migratoria manilensis. Biol. Chem. 2013, 394, 43-54. [CrossRef] [PubMed]

27. Sun, Y.L.; Huang, L.Q.; Pelosi, P.; Wang, C.Z. Expression in antennae and reproductive organs suggests a dual role of an odorant-binding protein in two sibling Helicoverpa species. PLoS ONE 2012, 7, e30040. [CrossRef] [PubMed]

28. Song, X.; Call, G.B.; Kirilly, D.; Xie, T. Notch signaling controls germline stem cell niche formation in the Drosophila ovary. Development 2007, 134, 1071-1080. [CrossRef] [PubMed]

29. Xu, J.; Gridley, T. Notch signaling during oogenesis in Drosophila melanogaster. Genet. Res. Int. 2012, 2012, 1-10. [CrossRef] [PubMed]

30. Rao, A.V.S.K.; Shaha, C. Role of glutathione S-transferases in oxidative stress-induced male germ cell apoptosis. Free Radic. Bio. Med. 2000, 29, 1015-1027. [CrossRef]

31. Talakhun, W.; Roytrakul, S.; Phaonakrop, N.; Kittisenachai, S.; Khamnamtong, B.; Klinbunga, S.; Menasveta, P. Identification of reproduction-related proteins and characterization of the protein disulfide isomerase A6 cDNA in ovaries of the giant tiger shrimp Penaeus monodon. Comp. Biochem. Physiol. Part D Genom. Proteom. 2012, 7, 180-190. [CrossRef] [PubMed]

32. Malone, C.D.; Brennecke, J.; Dus, M.; Stark, A.; McCombie, W.R.; Sachidanandam, R.; Hannon, G.J. Specialized piRNA pathways act in germline and somatic tissues of the Drosophila ovary. Cell 2009, 137, 522-535. [CrossRef] [PubMed]

33. Czech, B.; Preall, J.; Mcginn, J.; Hannon, G. A transcriptome-wide RNAi screen in the Drosophila ovary reveals factors of the germline piRNA pathway. Mol. Cell 2013, 50, 749-761. [CrossRef] [PubMed]

34. Peng, J.C.; Valouev, A.; Liu, N.; Lin, H. Piwi maintains germline stem cells and oogenesis in Drosophila through negative regulation of Polycomb Group proteins. Nat. Genet. 2016, 48, 283-291. [CrossRef] [PubMed]

35. Bardsley, A.; McDonald, K.; Boswell, R.E. Distribution of tudor protein in the Drosophila embryo suggests separation of functions based on site of localization. Development 1993, 119, 207-219. [PubMed]

36. Creed, T.M.; Loganathan, S.N.; Varonin, D.; Jackson, C.A.; Arkov, A.L. Novel role of specific Tudor domains in Tudor-Aubergine protein complex assembly and distribution during Drosophila oogenesis. Biochem. Bioph. Res. Commun. 2010, 402, 384-389. [CrossRef] [PubMed]

37. Dufourt, J.; Dennis, C.; Boivin, A.; Gueguen, N.; Théron, E.; Goriaux, C.; Pouchin, P.; Ronsseray, S.; Brasset, E.; Vaury, C. Spatio-temporal requirements for transposable element piRNA-mediated silencing during Drosophila oogenesis. Nucleic Acids Res. 2014, 42, 2512-2524. [CrossRef] [PubMed]

38. Richter, J.D.; Sonenberg, N. Regulation of cap-dependent translation by eIF4E inhibitory proteins. Nature 2005, 433, 477-480. [CrossRef] [PubMed]

39. Evsikov, A.V.; Graber, J.H.; Brockman, J.M.; Hampl, A.; Holbrook, A.E.; Singh, P.; Eppig, J.J.; Solter, D.; Knowles, B.B. Cracking the egg: Molecular dynamics and evolutionary aspects of the transition from the fully grown oocyte to embryo. Genes Dev. 2006, 20, 2713-2727. [CrossRef] [PubMed]

40. Piccioni, F.; Zappavigna, V.; Verrotti, A.C. Translational regulation during oogenesis and early development: The cap-poly(A) tail relationship. CR. Biol. 2005, 328, 863-881. [CrossRef] [PubMed]

41. Keyes, L.N.; Spradling, A.C. The Drosophila gene $f_{s}(2)$ cup interacts with otu to define a cytoplasmic pathway required for the structure and function of germ-line chromosomes. Development 1997, 124, 1419-1431. [PubMed] 
42. Zappavigna, V.; Piccioni, F.; Villaescusa, J.C.; Verrotti, A.C. Cup is a nucleocytoplasmic shuttling protein that interacts with the eukaryotic translation initiation factor $4 \mathrm{E}$ to modulate Drosophila ovary development. Proc. Natl. Acad. Sci. USA 2004, 101, 14800-14805. [CrossRef] [PubMed]

43. Xu, J.; Shu, J.; Zhang, Q. Expression of the Tribolium castaneum (Coleoptera: Tenebrionidae) hsp83 gene and its relation to oogenesis during ovarian maturation. J. Genet. Genom. 2010, 37, 513-522. [CrossRef]

44. Pisa, V.; Cozzolino, M.; Gargiulo, S.; Ottone, C.; Piccioni, F.; Monti, M.; Gigliotti, S.; Talamo, F.; Graziani, F.; Pucci, P. The molecular chaperone Hsp90 is a component of the cap-binding complex and interacts with the translational repressor Cup during Drosophila oogenesis. Gene 2009, 432, 67-74. [CrossRef] [PubMed]

45. Marin, R.; Tanguay, R.M. Stage-specific localization of the small heat shock protein Hsp27 during oogenesis in Drosophila melanogaster. Chromosoma 1996, 105, 142-149. [CrossRef] [PubMed]

46. Economou, K.; Kotsiliti, E.; Mintzas, A.C. Stage and cell-specific expression and intracellular localization of the small heat shock protein Hsp27 during oogenesis and spermatogenesis in the Mediterranean fruit fly, Ceratitis capitata. J. Insect Physiol. 2017, 96, 64-72. [CrossRef] [PubMed]

47. Li, Z.W.; Li, X.; Yu, Q.Y.; Xiang, Z.H.; Kishino, H.; Zhang, Z. The small heat shock protein (sHSP) genes in the silkworm, Bombyx mori, and comparative analysis with other insect sHSP genes. BMC Evol. Biol. 2009, 9, 215. [CrossRef] [PubMed]

48. Ádám, G.; Gausz, J.; Noselli, S.; Kurucz, É.; Andó, I.; Udvardy, A. Tissue- and developmental stage-specific changes in the subcellular localization of the $26 \mathrm{~S}$ proteasome in the ovary of Drosophila melanogaster. Gene Expr. Patterns 2004, 4, 329-333. [CrossRef] [PubMed]

49. Fredriksson, Å.; Johansson Krogh, E.; Hernebring, M.; Pettersson, E.; Javadi, A.; Almstedt, A.; Nyström, T. Effects of aging and reproduction on protein quality control in soma and gametes of Drosophila melanogaster. Aging Cell 2012, 11, 634-643. [CrossRef] [PubMed]

50. Tufail, M.; Takeda, M. Molecular characteristics of insect vitellogenins. J. Insect Physiol. 2008, 54, 1447-1458. [CrossRef] [PubMed]

51. Chen, S.L.; Lin, C.P.; Lu, K.H. cDNA isolation, expression, and hormonal regulation of yolk protein genes in the oriental fruit fly, Bactrocera dorsalis (Hendel) (Diptera: Tephritidae). J. Insect Physiol. 2012, 58, 763-770. [CrossRef] [PubMed]

52. Cingolani, G.; Petosa, C.; Weis, K.; Müller, C.W. Structure of importin- $\beta$ bound to the IBB domain of importin- $\alpha$. Nature 1999, 399, 221-229. [PubMed]

53. Lippai, M.; Tirián, L.; Boros, I.; Mihály, J.; Erdélyi, M.; Belecz, I.; Máthé, E.; Pósfai, J.; Nagy, A.; Udvardy, A. The Ketel gene encodes a Drosophila homologue of importin- $\beta$. Genetics 2000, 156, 1889-1900. [PubMed]

54. Máthé, E.; Bates, H.; Huikeshoven, H.; Deák, P.; Glover, D.M.; Cotterill, S. Importin- $\alpha 3$ is required at multiple stages of Drosophila development and has a role in the completion of oogenesis. Dev. Biol. 2000, 223, 307-322. [CrossRef] [PubMed]

55. Gorjánácz, M.; Adám, G.; Török, I.; Mechler, B.M.; Szlanka, T.; Kiss, I. Importin- $\alpha 2$ is critically required for the assembly of ring canals during Drosophila oogenesis. Dev. Biol. 2002, 251, 271-282. [CrossRef] [PubMed]

56. Mason, D.A.; Fleming, R.J.; Goldfarb, D.S. Drosophila melanogaster importin $\alpha 1$ and $\alpha 3$ can replace importin $\alpha 2$ during spermatogenesis but not oogenesis. Genetics 2002, 161, 157-170. [PubMed]

57. Ratan, R.; Mason, D.A.; Sinnot, B.; Goldfarb, D.S.; Fleming, R.J. Drosophila importin $\alpha 1$ performs paralog-specific functions essential for gametogenesis. Genetics 2008, 178, 839-850. [CrossRef] [PubMed]

58. Liu, Z.C.; Geisbrecht, E.R. "Importin" signaling roles for import proteins: The function of Drosophila importin-7 (DIM-7) in muscle-tendon signaling. Cell Adhes. Migr. 2012, 6, 1-4. [CrossRef] [PubMed]

59. Waring, G.L. Morphogenesis of the eggshells in Drosophila. Int. Rev. Cytol. 2000, 198, 67-108. [PubMed]

60. Cavaliere, V.; Bernardi, F.; Romani, P.; Duchi, S.; Gargiulo, G. Building up the Drosophila eggshell: First of all the eggshell genes must be transcribed. Dev. Dynam. 2008, 237, 2061-2072. [CrossRef] [PubMed]

61. Chen, A.; Gao, P.; Zhao, Q.; Tang, S.; Shen, X.; Zhang, G.; Qiu, Z.; Xia, D.; Huang, Y.; Xu, Y.; et al. Mutation of a vitelline membrane protein, BmEP80, is responsible for the silkworm "Ming" lethal egg mutant. Gene 2013, 515, 313-319. [CrossRef] [PubMed]

62. Sdralia, N.; Swevers, L.; Iatrou, K. BmVMP90, a large vitelline membrane protein of the domesticated silkmoth Bombyx mori, is an essential component of the developing ovarian follicle. Insect Biochem. Mol. Biol. 2012, 42, 717-727. [CrossRef] [PubMed] 
63. Kendirgi, F.; Swevers, L.; Iatrou, K. An ovarian follicular epithelium protein of the silkworm (Bombyx mori) that associates with the vitelline membrane and contributes to the structural integrity of the follicle. FEBS Lett. 2002, 524, 59-68. [CrossRef]

64. Velentzas, A.D.; Velentzas, P.D.; Sagioglou, N.E.; Konstantakou, E.G.; Anagnostopoulos, A.K.; Tsioka, M.M.; Mpakou, V.E.; Kollia, Z.; Consoulas, C.; Margaritis, L.H. Targeted downregulation of s36 protein unearths its cardinal role in chorion biogenesis and architecture during Drosophila melanogaster oogenesis. Sci. Rep. 2016, 6, 35511. [CrossRef] [PubMed]

65. Konstandi, O.A.; Papassideri, I.S.; Stravopodis, D.J.; Kenoutis, C.A.; Hasan, Z.; Katsorchis, T.; Wever, R.; Margaritis, L.H. The enzymatic component of Drosophila melanogaster chorion is the Pxd peroxidase. Insect Biochem. Mol. Biol. 2005, 35, 1043-1057. [CrossRef] [PubMed]

66. Konstandi, O.A.; Papassideri, I.S.; Stravopodis, D.J.; Antonelou, M.H.; Kenoutis, C.A.; Stefanidou, D.C.; Margaritis, L.H. The dual role of chorion peroxidase in Bactrocera oleae chorion assembly. Int. J. Dev. Biol. 2006, 50, 543-552. [CrossRef] [PubMed]

67. Buszczak, M.; Freeman, M.R.; Carlson, J.R.; Bender, M.; Cooley, L.; Segraves, W.A. Ecdysone response genes govern egg chamber development during mid-oogenesis in Drosophila. Development 1999, 126, 4581-4589. [PubMed]

68. Bernardi, F.; Romani, P.; Tzertzinis, G.; Gargiulo, G.; Cavaliere, V. EcR-B1 and Usp nuclear hormone receptors regulate expression of the VM32E eggshell gene during Drosophila oogenesis. Dev. Biol. 2009, 328, 541-551. [CrossRef] [PubMed]

69. Xu, K.K.; Yang, W.J.; Tian, Y.; Wu, Y.B.; Wang, J.J. Insulin signaling pathway in the oriental fruit fly: The role of insulin receptor substrate in ovarian development. Gen. Comp. Endocr. 2015, 126, 125-133. [CrossRef] [PubMed]

70. Abrisqueta, M.; Sueren-Castillo, S.; Maestro, J.L. Insulin receptor-mediated nutritional signalling regulates juvenile hormone biosynthesis and vitellogenin production in the German cockroach. Insect Biochem. Mol. Biol. 2014, 49, 14-23. [CrossRef] [PubMed]

71. Vanorny, D.A.; Prasasya, R.D.; Chalpe, A.J.; Kilen, S.M.; Mayo, K.E. Notch signaling regulates ovarian follicle formation and coordinates follicular growth. Mol. Endocrinol. 2014, 28, 499-511. [CrossRef] [PubMed]

72. Bradford, M.M. A rapid and sensitive method for the quantitation of microgram quantities of protein utilizing the principle of protein-dye binding. Anal. Biochem. 1976, 72, 248-254. [CrossRef]

73. Shilov, I.V.; Seymour, S.L.; Patel, A.A.; Loboda, A.; Tang, W.H.; Keating, S.P.; Hunter, C.L.; Nuwaysir, L.M.; Schaeffer, D.A. The paragon algorithm, a next generation search engine that uses sequence temperature values and feature probabilities to identify peptides from tandem mass spectra. Mol. Cell. Proteom. 2007, 6, 1638-1655. [CrossRef] [PubMed]

74. Conesa, A.; Götz, S.; García-Gómez, J.M.; Terol, J.; Talón, M.; Robles, M. Blast2GO: A universal tool for annotation, visualization and analysis in functional genomics research. Bioinformatics 2005, 21, 3674-3676. [CrossRef] [PubMed]

75. Shen, G.M.; Jiang, H.B.; Wang, X.N.; Wang, J.J. Evaluation of endogenous references for gene expression profiling in different tissues of the oriental fruit fly Bactrocera dorsalis (Diptera: Tephritidae). BMC Mol. Biol. 2010, 11, 76. [CrossRef] [PubMed]

(C) 2017 by the authors. Licensee MDPI, Basel, Switzerland. This article is an open access article distributed under the terms and conditions of the Creative Commons Attribution (CC BY) license (http://creativecommons.org/licenses/by/4.0/). 